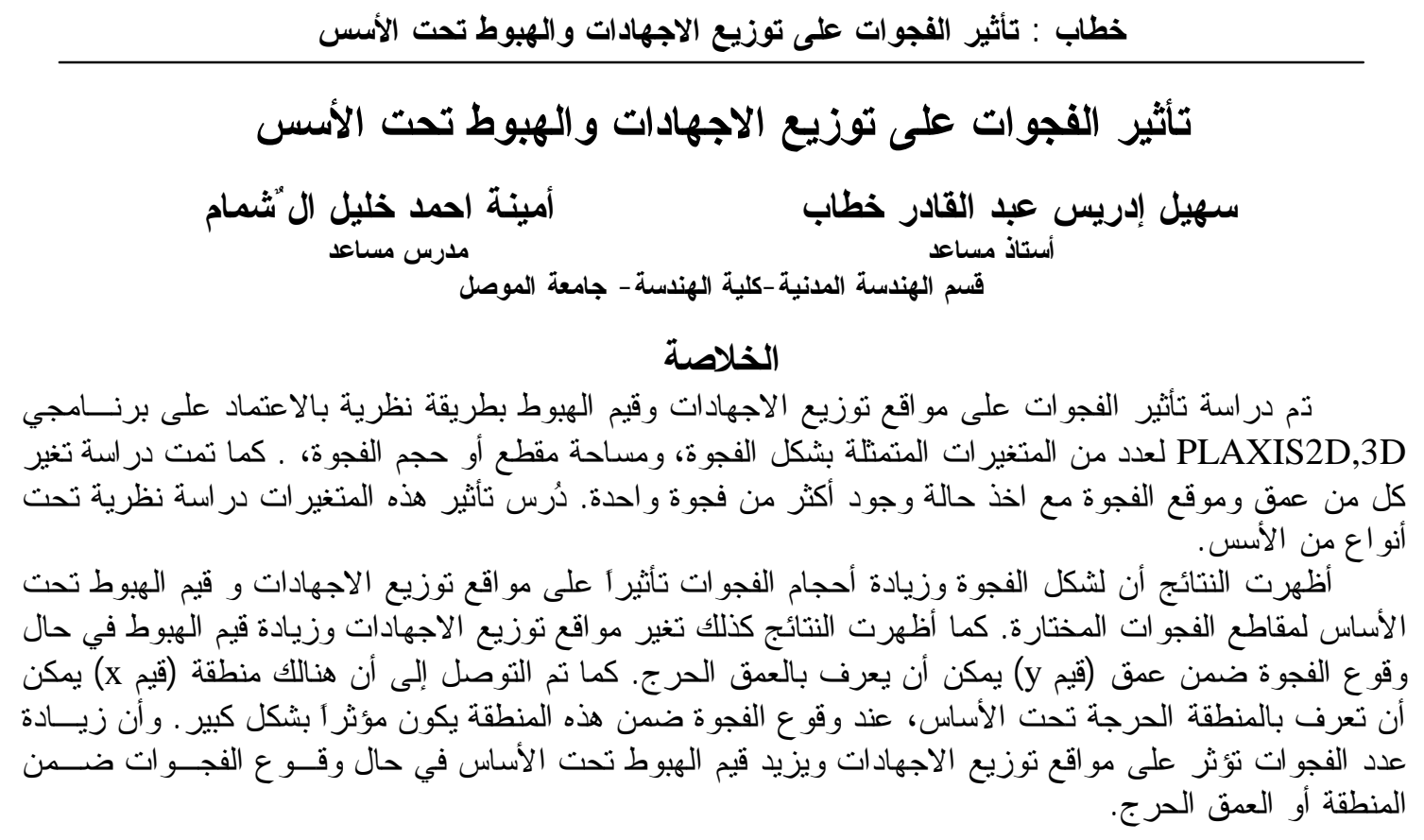

\title{
Effect of cavity on Stress distribution and Settlement under Foundation
}

\section{S. A. Khattab \\ Assistant Professor}

A. A. Khalil

Assistant Lecturer

Civil Engineering Department-MOSUL University

\begin{abstract}
In this research the effect of cavities on the stability of foundation has been studied using non-linear finite elements analysis through the programs PLAXIS2D, and PLAXIS3D. The study included a number of variables represented by shape, size, sectional area, location, and depth of a single cavity under the base of footings. The effect of the above variables on settlement and stress distribution was studied on isolated square, round and strip footing.

Results showed that the shape and volume of the cavity has an effect on the settlement and concentration of stress under the footing for the chosen cavity sections (Circular, Ellipse1, Ellipes2, Loaf, Square) when the cavity is situated at a depth less than twice the width of strip foundation or 1.5 times the width/diameter of isolated square/circular footings. The study also showed an increase in the values of settlement and concentration of stress at what may be termed as the critical depth under the footing. It was also concluded that there exists a zone that may be called the critical zone below the footing (zone of radial shear and failure plane). If a cavity is situated within this zone then it will have a serious effect. Large value of settlement were recorded for cavities situated within this zone under the footing.
\end{abstract}

Keywords: Cavity, Settlement, Stress, Finite Element. 


\section{Al-Rafidain Engineering $\quad$ Vol.17 $\quad$ No.6 $\quad$ Dec. 2009}

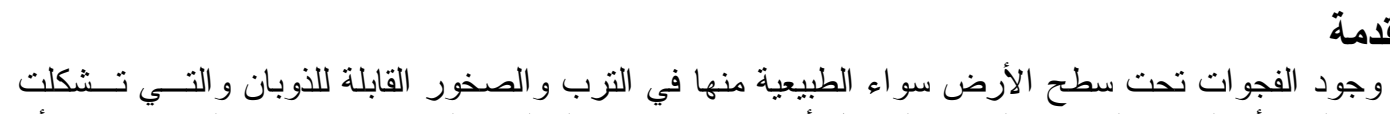

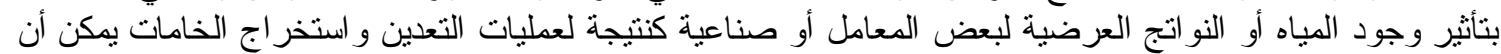

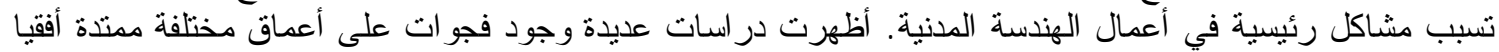

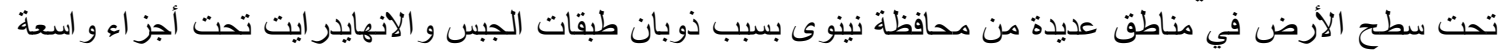

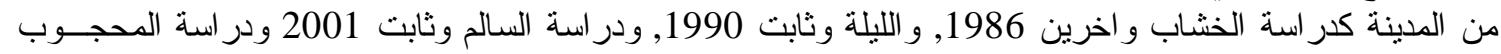

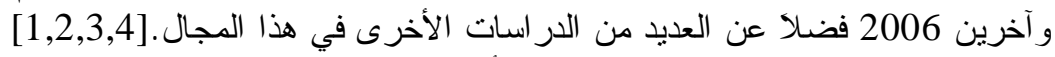

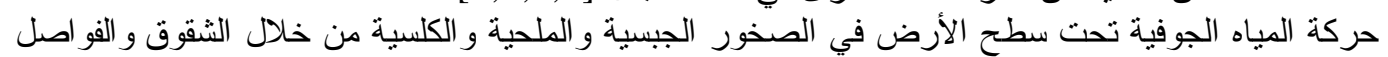

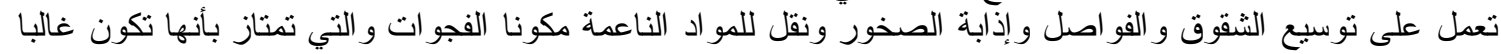

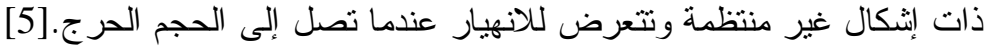

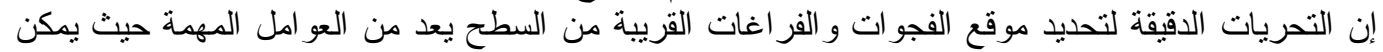

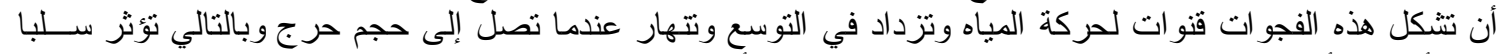

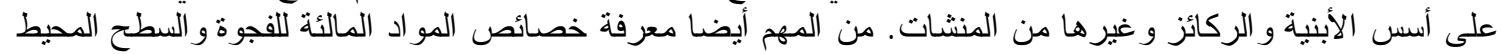

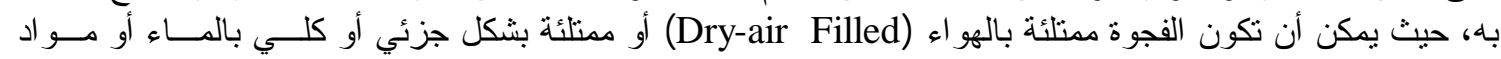

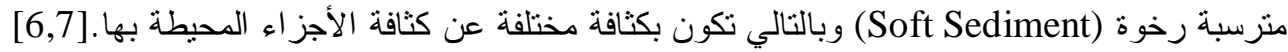

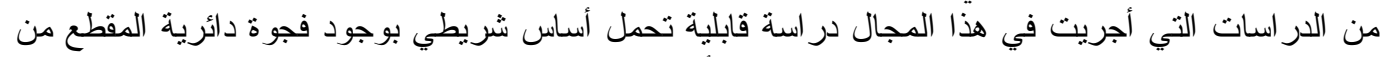

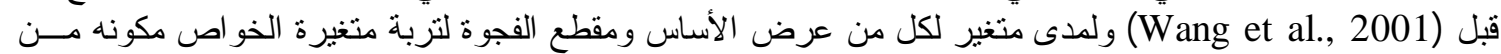

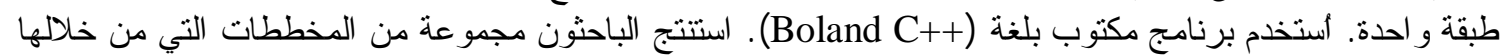

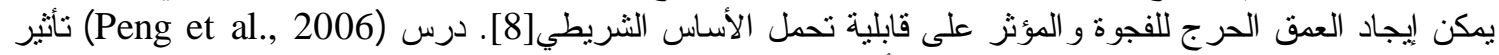

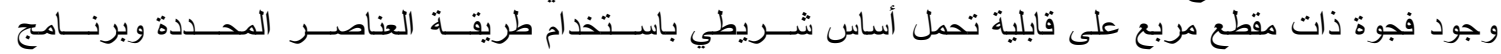
PLAXIS2D

[9] الأساس ويقلل من قابلية تحمله الباحثة

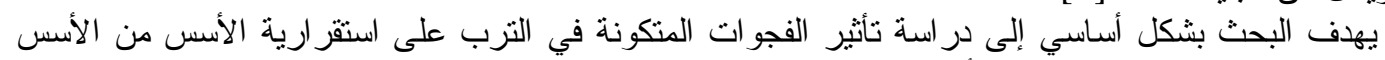

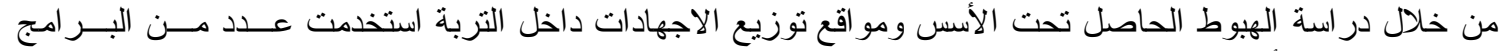

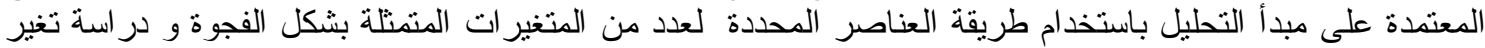

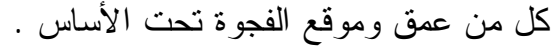

\section{Constitutive Relationships العلاقات التكوينية 2}

\section{Soil Model تمثيل التربة}

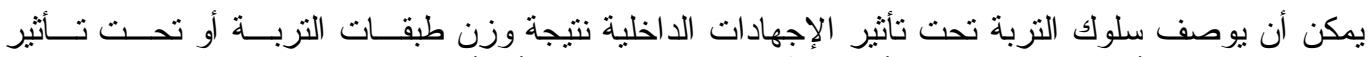

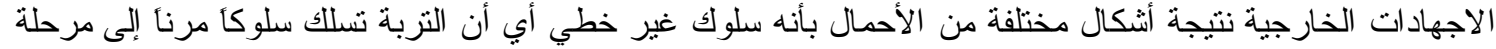

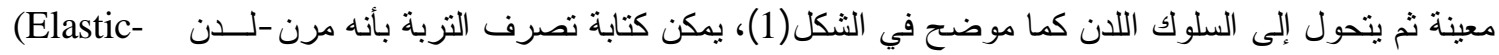
Plastic)

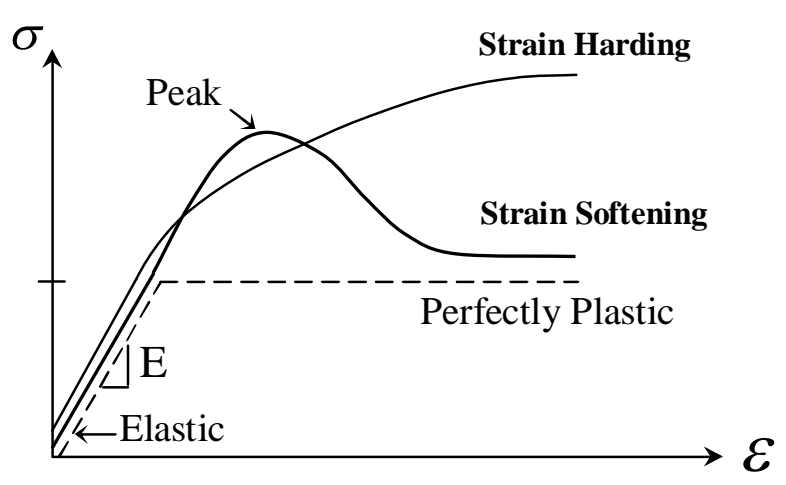

الثكل(1): علاقة الإجهاد - الانفعال في التربة [10] 


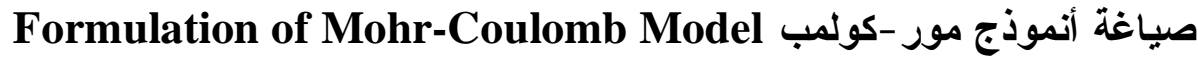

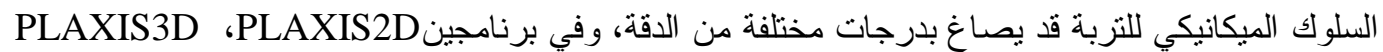

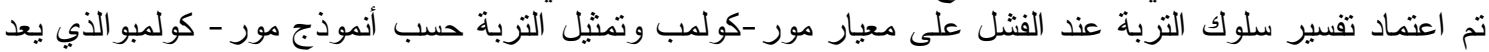

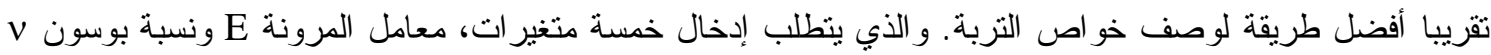

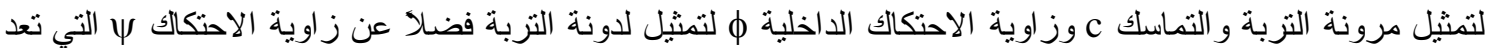

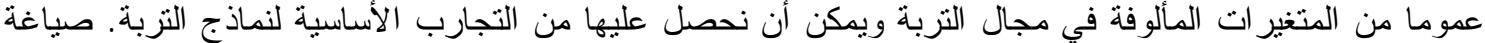

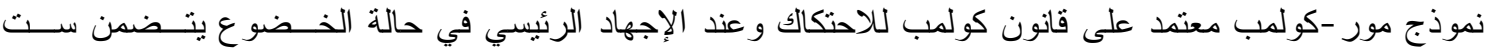

$$
\left.\begin{array}{l}
f_{1 a}=\frac{1}{2}\left(\sigma_{2}^{\prime}-\sigma_{3}^{\prime}\right)+\frac{1}{2}\left(\sigma_{2}^{\prime}+\sigma_{3}^{\prime}\right) \sin \phi-c \cos \varphi \leq 0 \\
f_{1 b}=\frac{1}{2}\left(\sigma_{3}^{\prime}-\sigma_{2}^{\prime}\right)+\frac{1}{2}\left(\sigma_{3}^{\prime}+\sigma_{2}^{\prime}\right) \sin \phi-c \cos \varphi \leq 0 \\
f_{2 a}=\frac{1}{2}\left(\sigma_{3}^{\prime}-\sigma_{1}^{\prime}\right)+\frac{1}{2}\left(\sigma_{3}^{\prime}+\sigma_{1}^{\prime}\right) \sin \phi-c \cos \varphi \leq 0 \\
f_{2 b}=\frac{1}{2}\left(\sigma_{1}^{\prime}-\sigma_{3}^{\prime}\right)+\frac{1}{2}\left(\sigma_{1}^{\prime}+\sigma_{3}^{\prime}\right) \sin \phi-c \cos \varphi \leq 0 \\
f_{3 a}=\frac{1}{2}\left(\sigma_{1}^{\prime}-\sigma_{2}^{\prime}\right)+\frac{1}{2}\left(\sigma_{1}^{\prime}+\sigma_{2}^{\prime}\right) \sin \phi-c \cos \varphi \leq 0 \\
f_{3 b}=\frac{1}{2}\left(\sigma_{2}^{\prime}-\sigma_{1}^{\prime}\right)+\frac{1}{2}\left(\sigma_{2}+\sigma_{1}\right) \sin \phi-c \cos \varphi \leq 0
\end{array}\right\}
$$

معادلات خضو عور: [11,12]

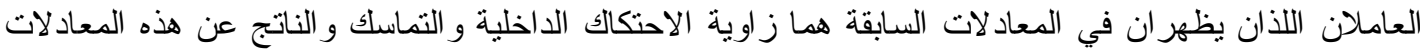

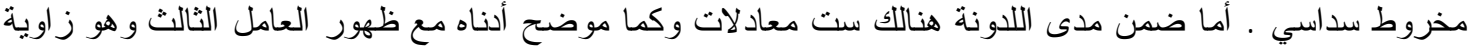

$$
\begin{aligned}
& g_{1 a}=\frac{1}{2}\left(\sigma_{2}^{\prime}-\sigma_{3}^{\prime}\right)+\frac{1}{2}\left(\sigma_{2}^{\prime}+\sigma_{3}^{\prime}\right) \sin \psi \\
& g_{1 b}=\frac{1}{2}\left(\sigma_{3}^{\prime}-\sigma_{2}^{\prime}\right)+\frac{1}{2}\left(\sigma_{3}^{\prime}+\sigma_{2}^{\prime}\right) \sin \psi \\
& g_{2 a}=\frac{1}{2}\left(\sigma_{3}^{\prime}-\sigma_{1}^{\prime}\right)+\frac{1}{2}\left(\sigma_{3}^{\prime}+\sigma_{1}^{\prime}\right) \sin \psi \\
& g_{2 b}=\frac{1}{2}\left(\sigma_{1}^{\prime}-\sigma_{3}^{\prime}\right)+\frac{1}{2}\left(\sigma_{1}^{\prime}+\sigma_{3}^{\prime}\right) \sin \psi \\
& g_{3 a}=\frac{1}{2}\left(\sigma_{1}^{\prime}-\sigma_{2}^{\prime}\right)+\frac{1}{2}\left(\sigma_{1}^{\prime}+\sigma_{2}^{\prime}\right) \sin \psi \\
& g_{3 b}=\frac{1}{2}\left(\sigma_{2}^{\prime}-\sigma_{1}\right)+\frac{1}{2}\left(\sigma_{2}+\sigma_{1}^{\prime}\right) \sin \psi
\end{aligned}
$$

الاحتكاك

\section{Elements Used to Model Soil العناصر المستخدمة لتمثيل التربة}

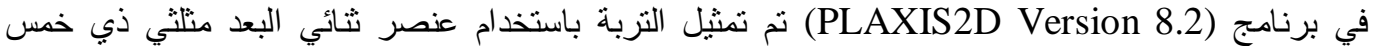

عشرة عقدة (15-Node Triangles Element) و اثثني عشرة نقطة فئنة إجهاد (12-Stress or Gauss Points)

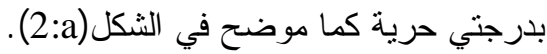
إما في برنامج (PLAXIS3D Tunnel Version 1.2) تم تمثيل التزبة باستخدام عنصر ثلاثي البعد ذي في

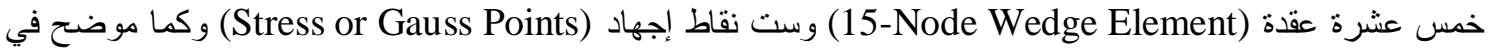
الثكل(b:).نم اختيار حجم العنصر المناسب في الحل بعد عدد من المحاو لات مع مر اعاة عمل تتعيم للشبكة تحت

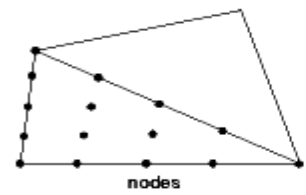

(a):2D Flement

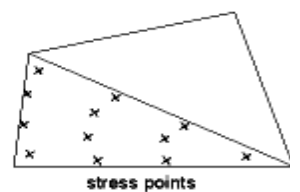

11,12].

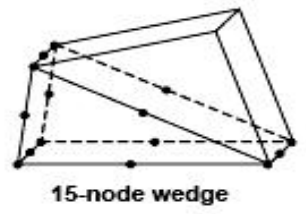

(h): 3D Element الأساس وحول الفجوة.

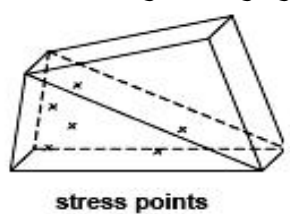

stress points 
3 - النتائج والمنـاقشة أساسبين هما: (أولا): در اسة تأثير الفجوات على توزيع الاجهادات و الهيوط تحت أساس شريطي.

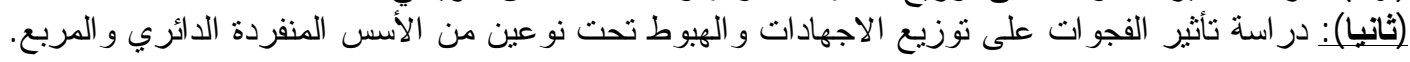

$$
\text { تم الأخذ بنظر الاعتبار المتغير ات الآتية في الدر اسة. }
$$

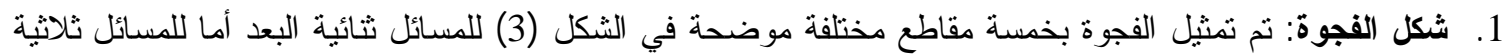

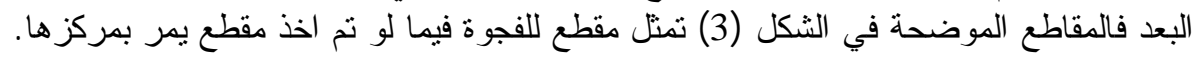

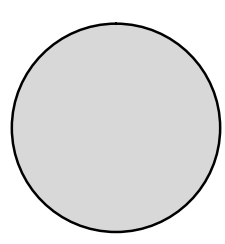

دائري

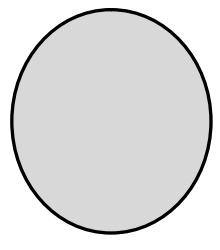

بيضوي 1

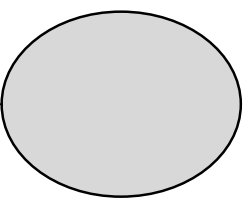

بيضوي2

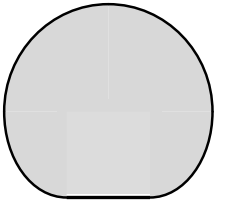

لوف

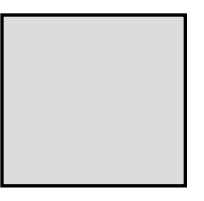

مربع

الثكل(3):مخطط لأشكال مقاطع الفجو ات المستخدمة. [13]

2. عمق الفجوة: المقصود بعمق الفجوة هو بعد مركز الفجوة عن قاعدة الأساس و الذي تم تعريفه في المسائل المطبقة

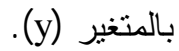

3. موقع الفجوة: المقصود بموقع الفجوة هو بعد مركز الفجوة عن مركز الأساس(Center Line) و الذي تم تعريفه في

المسائل المطبقة بالمتغير (x).

4. عدد الفجوات: تم اخذ حالة وجود أكثر من فجوة واحدة حيث أن اغلب الدراسات والبحوث تفرض حالة وجود فجوة و احدة أثناء الدر اسة.

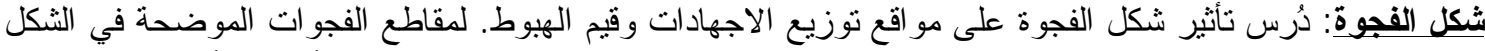

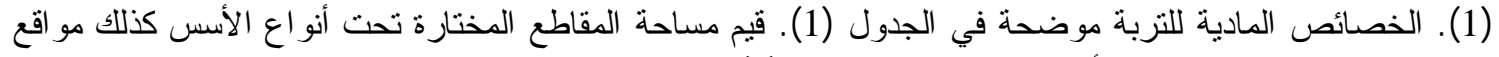

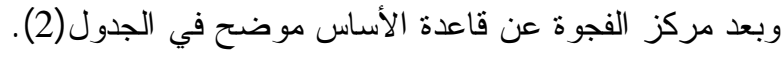

\section{الجدول(1): الخصائص المادية للتربة المستخدمة في دراسة}

\begin{tabular}{||l|c|c|c|c||}
\hline \multicolumn{1}{|c|}{ Parameter } & \multirow{2}{*}{ Name } & \multirow{2}{*}{ Unite } & \multicolumn{2}{c||}{ Value } \\
\cline { 4 - 5 } & & & $\mathrm{A}$ & $\mathrm{B}$ \\
\hline $\begin{array}{l}\text { Soil unit weight above } \\
\text { phreatic level }\end{array}$ & $\gamma_{\text {unsat. }}$ & $\mathrm{kN} / \mathrm{m}^{3}$ & 16.5 & 17 \\
\hline $\begin{array}{l}\text { Soil unit weight under } \\
\text { phreatic level }\end{array}$ & $\gamma_{\text {sat. }}$ & $\mathrm{kN} / \mathrm{m}^{3}$ & 20 & 20 \\
\hline Coefficient of permeability & $\mathrm{K}$ & $\mathrm{m} / \mathrm{day}$ & $8.5^{*} 10^{-5}$ & $8.5 * 10^{-5}$ \\
\hline Young's modulus & $\mathrm{E}$ & $\mathrm{kN} / \mathrm{m}^{2}$ & 5000 & 15000 \\
\hline Poisson's ratio & $v$ & - & 0.35 & 0.3 \\
\hline Cohesion & $\mathrm{c}$ & $\mathrm{kN} / \mathrm{m}^{2}$ & 17.8 & 25 \\
\hline Angle of internal friction & $\phi$ & Deg. & 29 & 29 \\
\hline
\end{tabular}




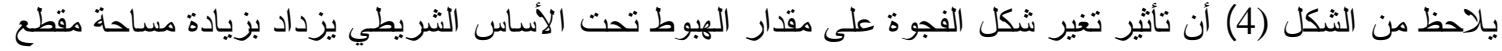

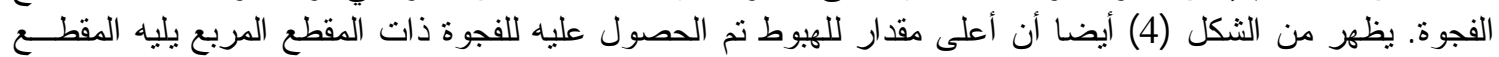

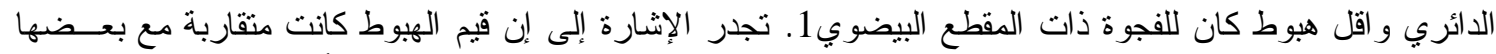
للفجوات ذات المقاطع الدائرية و المربعة من جهة وذات المقاط المقع البيضوي2 و اللوف من جهة أخرى.
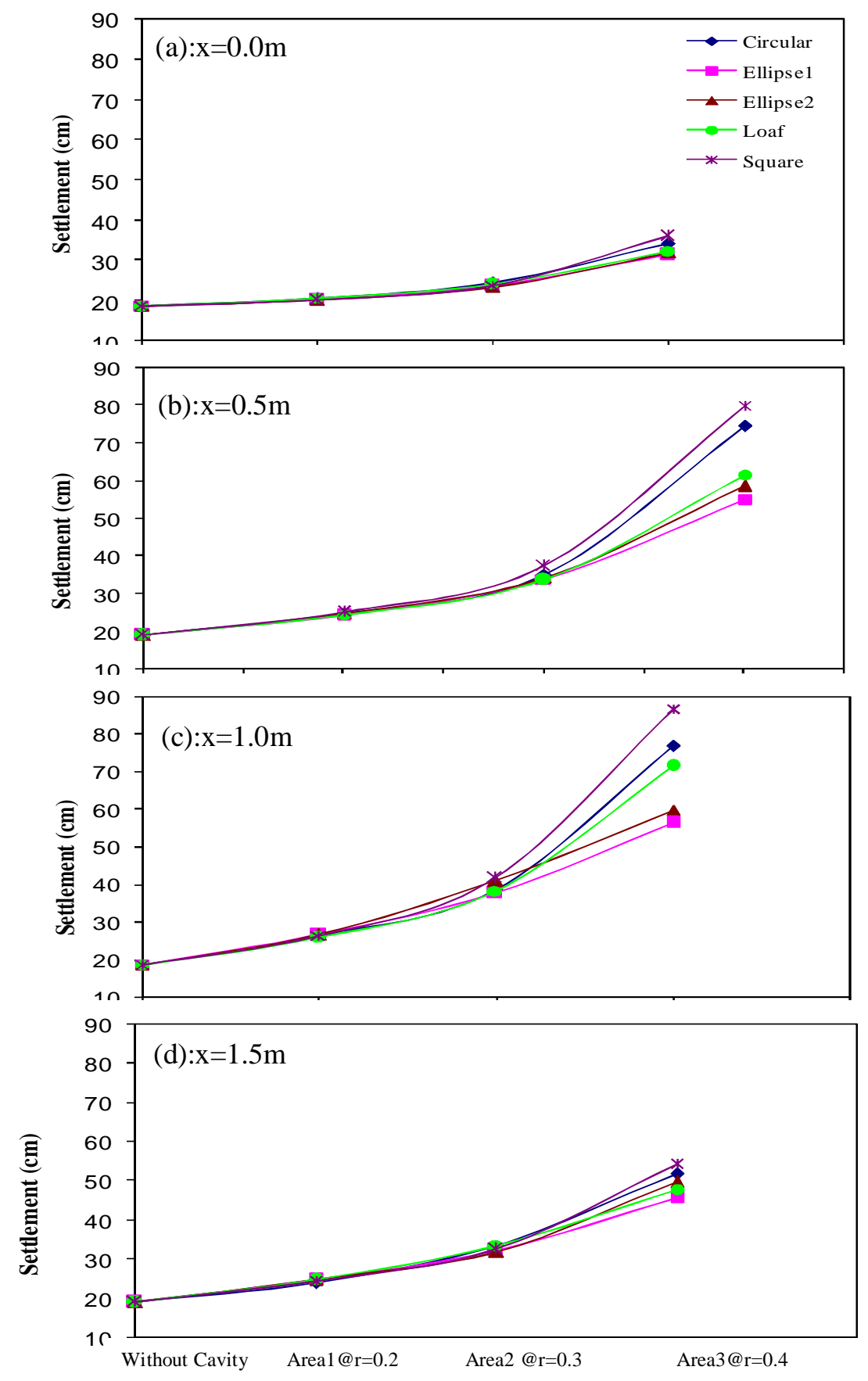

Area of cavity $\left(\mathbf{m}^{2}\right)$

الثكل(4):العلاقة بين الهبوط ومساحة مقطع الفجوة عند (a) (a)

(d):x=1.5m, (c):x=1.0m, (b):x=0.5m, (a):x=0m 
$\begin{array}{llll}\text { Al-Rafidain Engineering } & \text { Vol.17 } & \text { No.6 } & \text { Dec. } 2009\end{array}$

\begin{tabular}{|c|c|c|c|c|c|c|}
\hline$\tilde{g}$ & 岁 & $\tilde{y}$ & $\tilde{\sigma}$ & Нू & $\tilde{y}$ & E \\
\hline 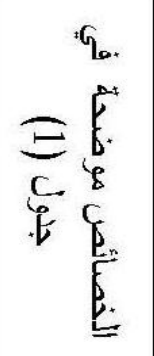 & 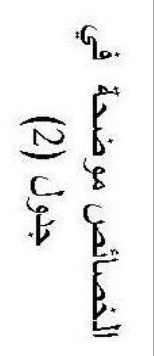 & 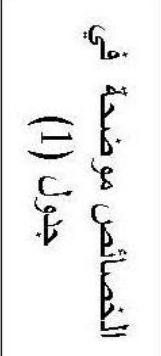 & 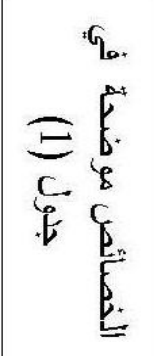 & $\begin{array}{rl} & 6 . \\
E & E \\
C & E \\
E & E \\
E & E \\
E\end{array}$ & 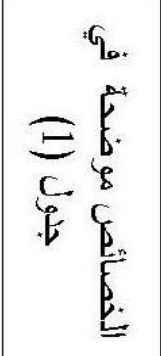 & 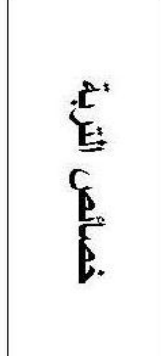 \\
\hline $\begin{array}{l}5 \\
N \\
\omega \\
+\infty \\
\omega \\
0\end{array}$ & & 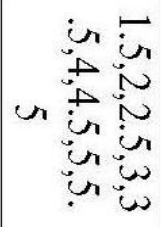 & $\begin{array}{l}N \\
w \\
u \\
u \\
0 \\
\vdots \\
\infty\end{array}$ & 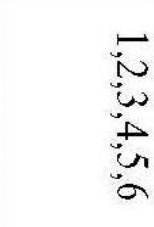 & $i$ & \\
\hline$\frac{N}{0}$ & 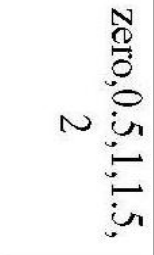 & $\frac{N}{0}$ & N & $\frac{N}{0}$ & 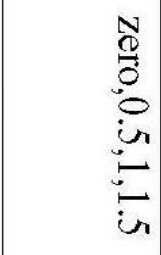 & $\frac{\underline{E}}{E}$ \\
\hline i & is & $i_{i}^{0} \underset{i}{0}$ & in $\stackrel{\circ}{\circ}$ i & $\stackrel{0}{+} \underset{i}{0}$ & $\stackrel{P}{P}$ is & 䓃象 \\
\hline$\prod_{\omega}^{\varpi}$ & $\underset{N}{\mathbb{N}}$ & $\prod_{\omega}^{\oplus}$ & $\prod_{\omega}^{\infty}$ & $\underset{\omega}{\|}$ & $\prod_{\omega}$ & $\widehat{E}_{\underline{E}}^{E} E$ \\
\hline$\frac{\tilde{E}^{2}}{c}$ & E. & 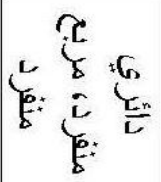 & E. & 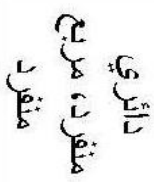 & E. & ${ }_{\Sigma}^{E} m$. \\
\hline 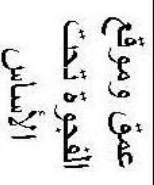 & 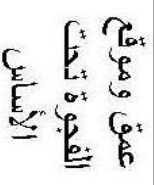 & $\frac{e_{0}^{*}}{e_{0}^{*}}$ & 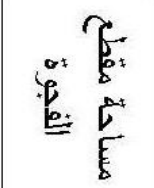 & $\frac{e_{0}^{*}}{e_{n}^{e}}$ & $\frac{\dot{e}}{\tilde{E}}$ & $\frac{E}{E}$ \\
\hline$\omega$ & $\Theta$ & $\omega$ & $\Theta$ & $\omega$ & $\Theta$ & $E_{E} m$. \\
\hline
\end{tabular}


خطاب : تأثثر الفجوات على توزيع الاجهادات والهجوط تحت الأسس

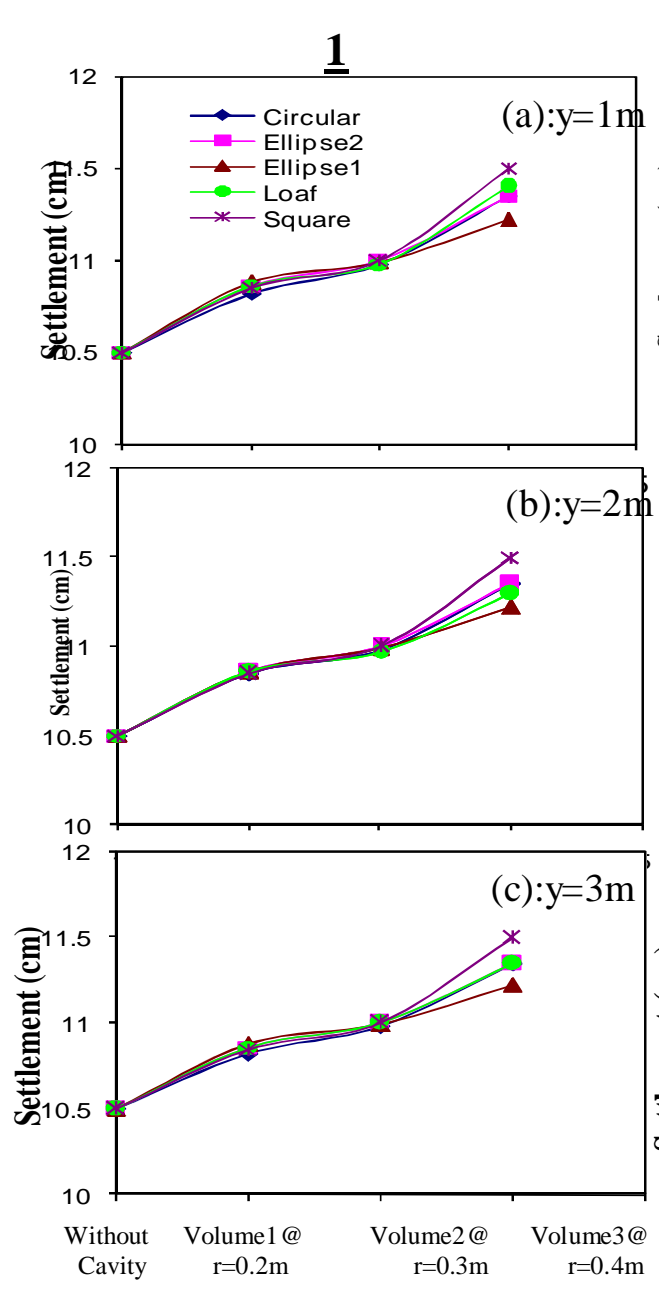

Volume of cavity $\left(\mathrm{m}^{3}\right)$

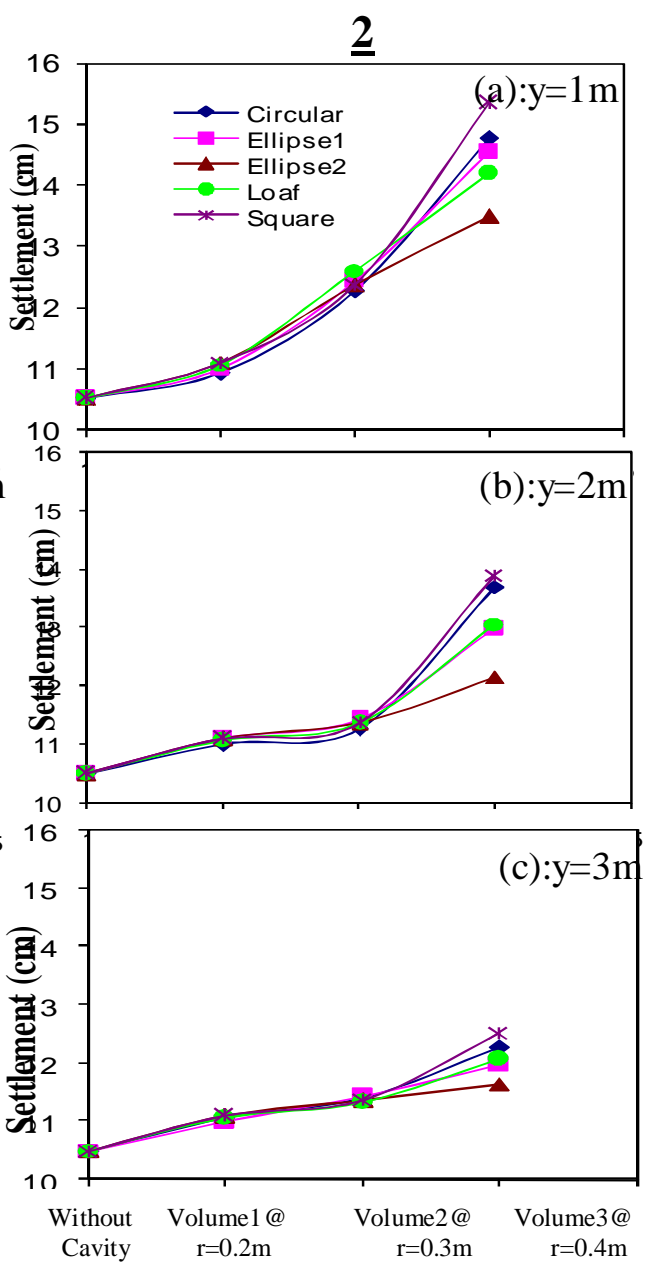

Volume of cavity $\left(\mathrm{m}^{3}\right)$

(c):y=3m و و و (a):y=1m (a):y=2m الثنكل(5): العلاقة بين الهبوط وحجم الفجوة عند

يلاحظ من الأثكال (5:1) و (5:2) أن مقدار الهبوط تحت الأساسين المنفردين دائري و المربع يزداد بزيادة

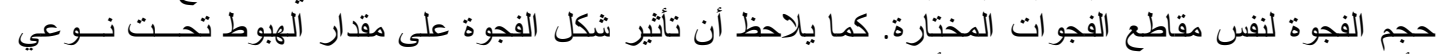

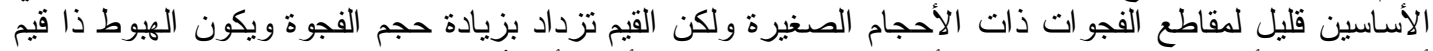

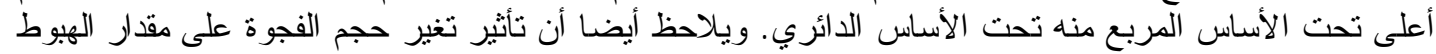

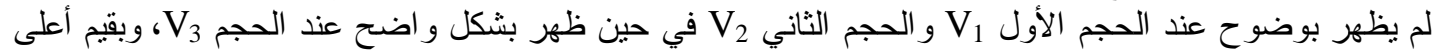

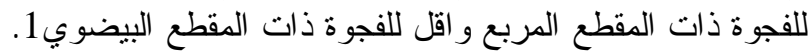

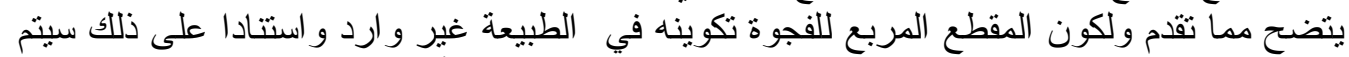
تمثيل الفجوة بمقطع دائري لدر اسة باقي المتغير ات كون المقات الفعع المربع الأكثر هبوطا.

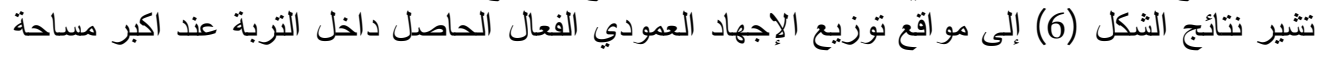

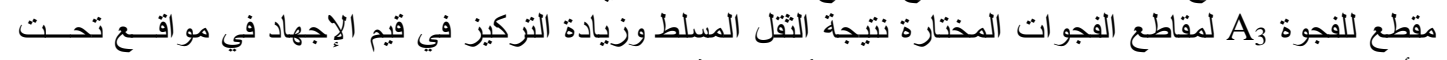

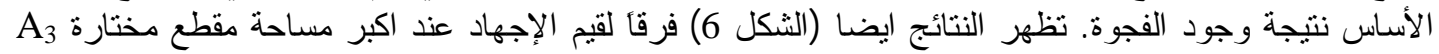

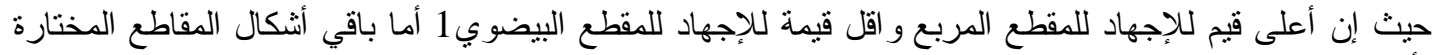

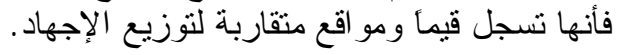




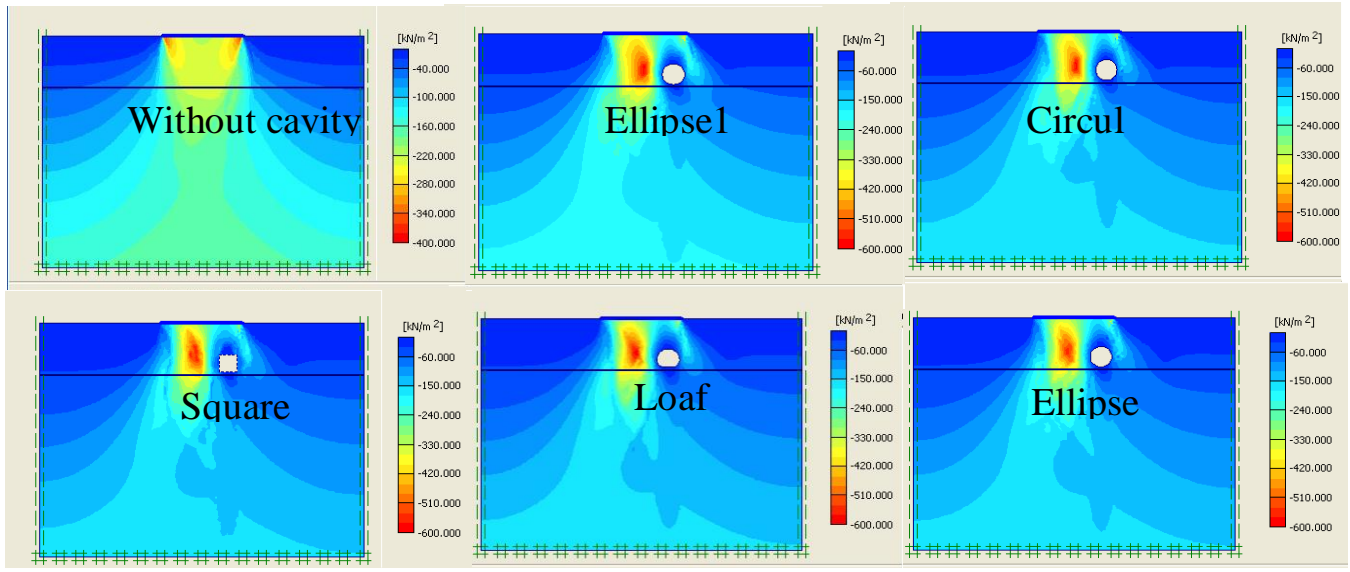

الثكل(6): تأثبر تغير شكل مقطع الفجوة على شدة توزيع الإجهاد في التربة (لقيمة

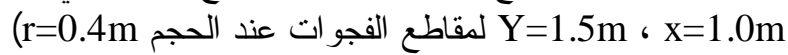

يبين الثكل (7) تأثير وجود وتغير شكل ومساحة مقطع الفجوة على توزيع الإجهاد العمودي الفعال داخل

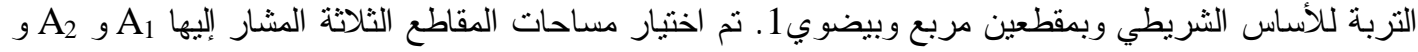

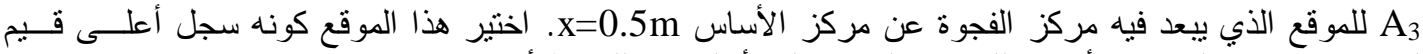

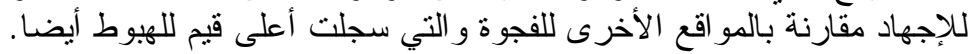

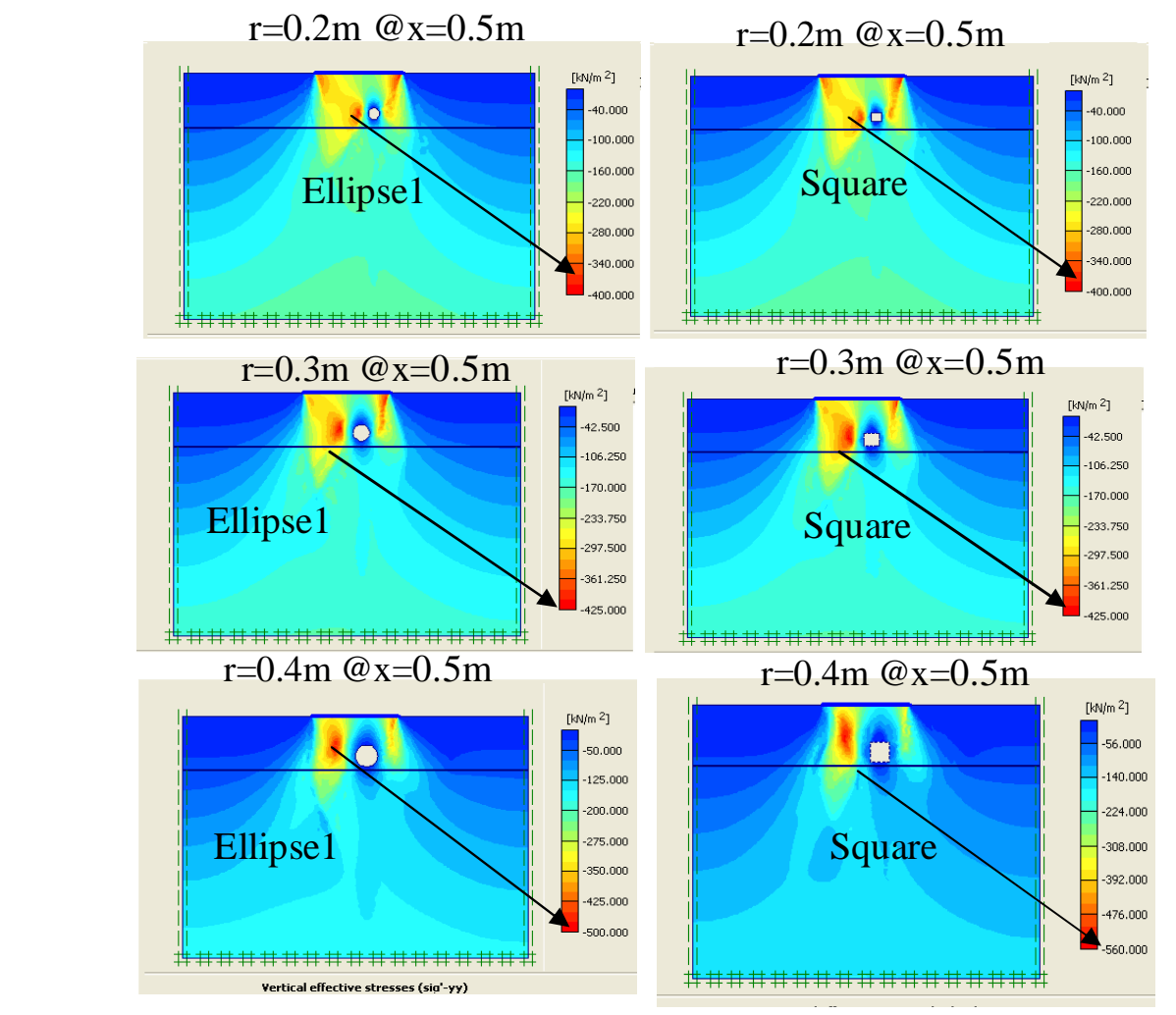
$\mathrm{r}=0.2 \mathrm{~m} @ \mathrm{x}=0.5 \mathrm{~m}$

الثكل(7): تأثير تغير شكل مقطع الفجوة على شدة نوزيع الإجهاد في التربة (لبعد مركز الفجوة عن

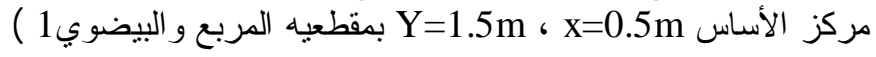


خطاب : تأثثر الفجوات على توزيع الاجهادات والهجوط تحت الأسس
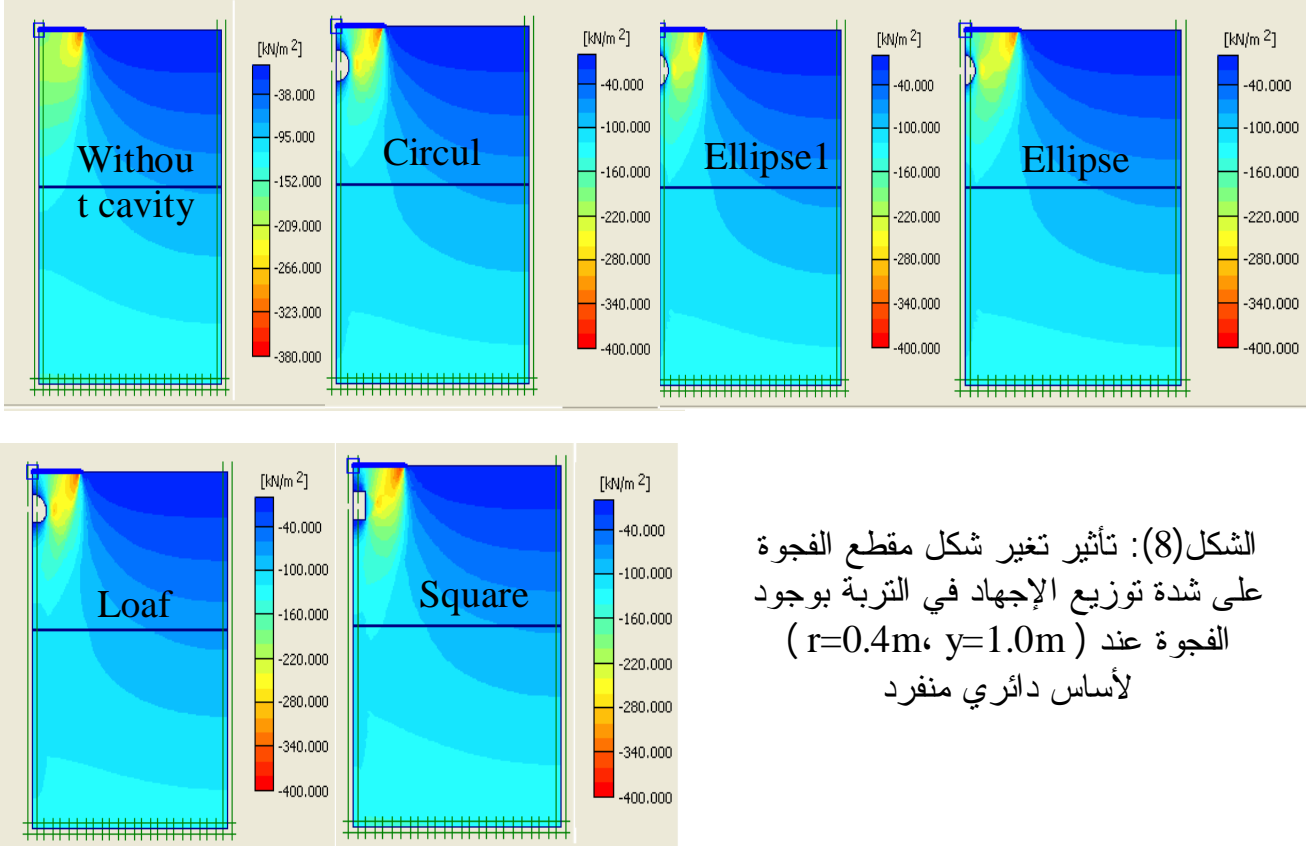

الثكل(8): تأثير تغير شكل مقطع الفجوة

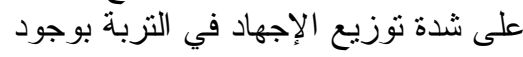

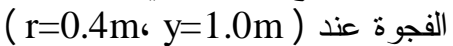

لأساس دائري منفرد

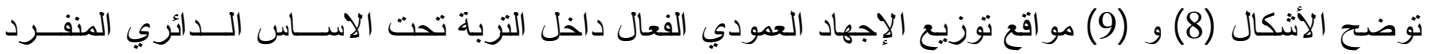

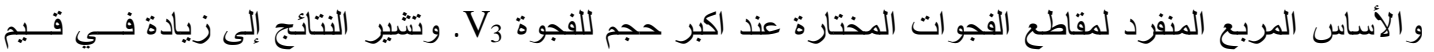

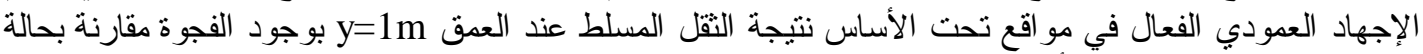

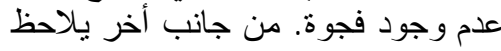

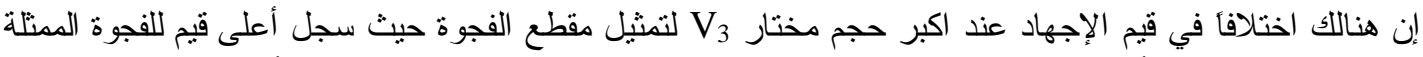

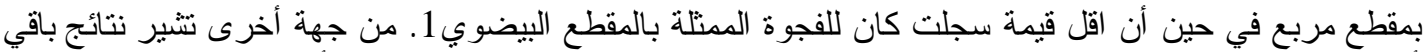

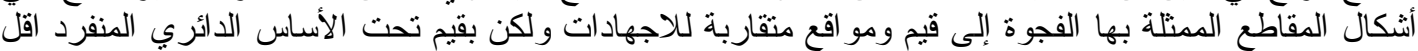
منه تحت الأساس المربع المنفرد.

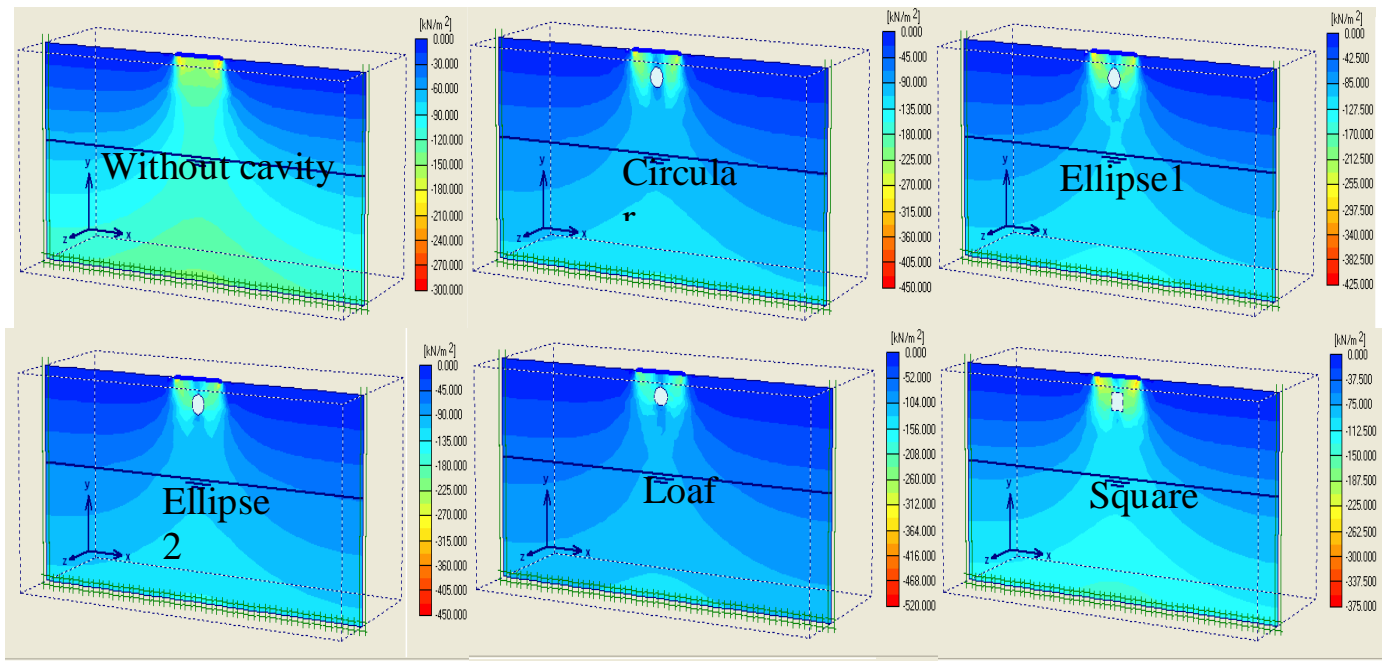

الثكل(9): تأثير تغير شكل مقطع الفجوة على شدة توزيع الإجهاد في التزبة بوجود الفجوة

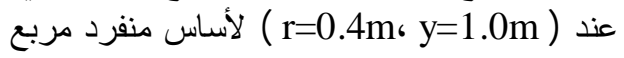




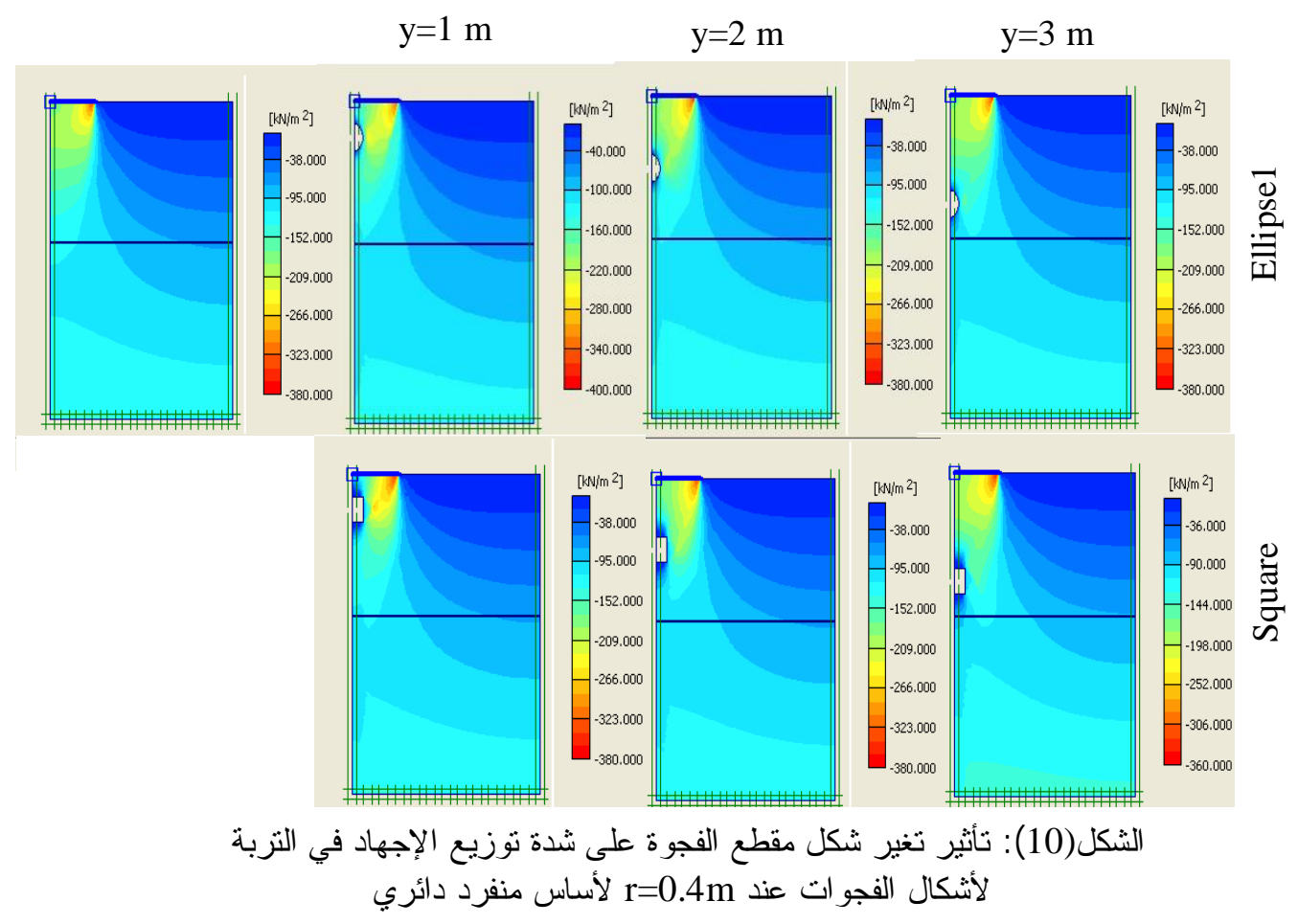

توضح الأثكال (10) و (11) توزيع الإجهاد العمودي الفعال داخل النزبة تحت الأساس الدائري المنفرد و المربع

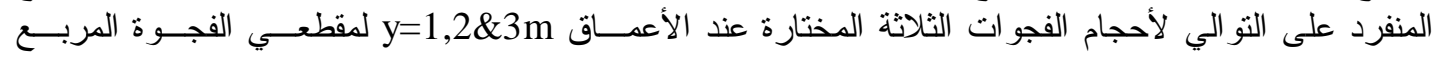

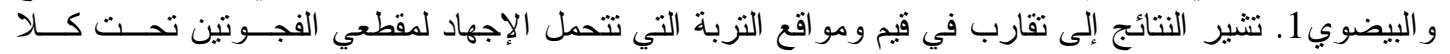

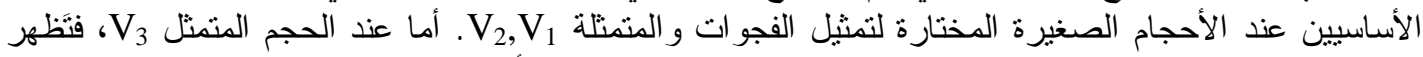

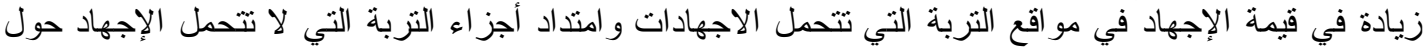

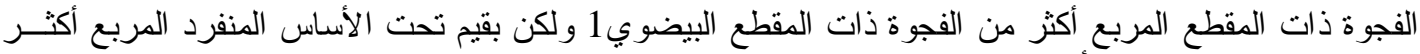
بقليل من تلك القيم تحت الأساس الدائري المنفرد.

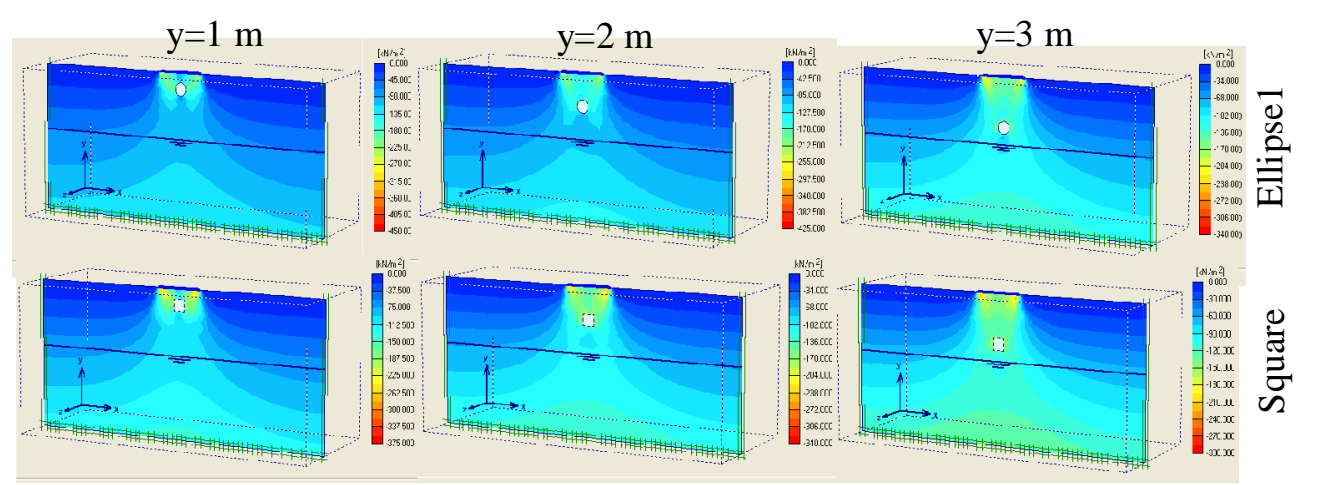

الثكل(11): تأثثر تغير شكل مقطع الفجوة على توزيع الإجهاد في التزبة

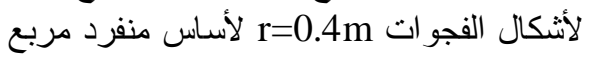

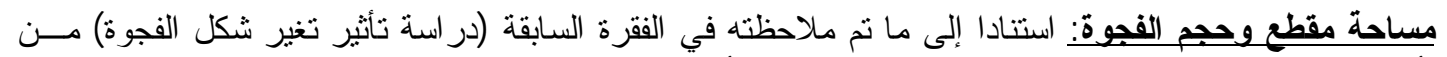

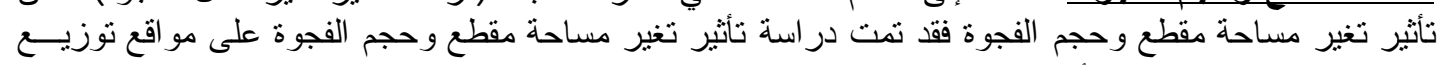

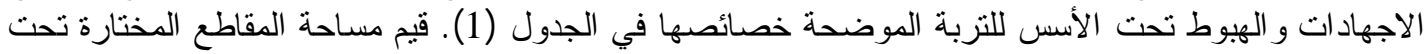

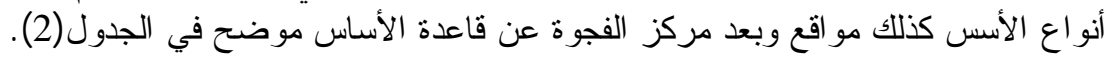


يبين الثكل(12) العلاقة بين إحداثبات (x,y) الفجوة مع الهبوط تحت الأساس لمقطع الفجوة ولــنالاث مسـساحات

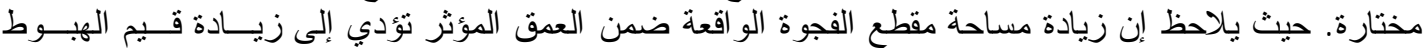

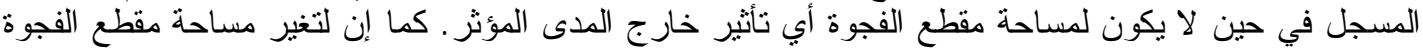

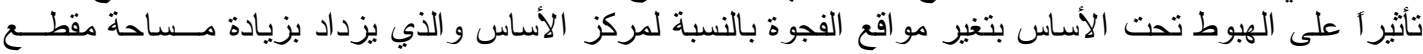

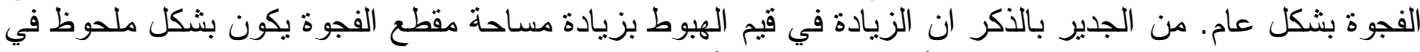

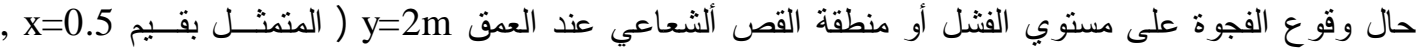
الموضح في المخططات.
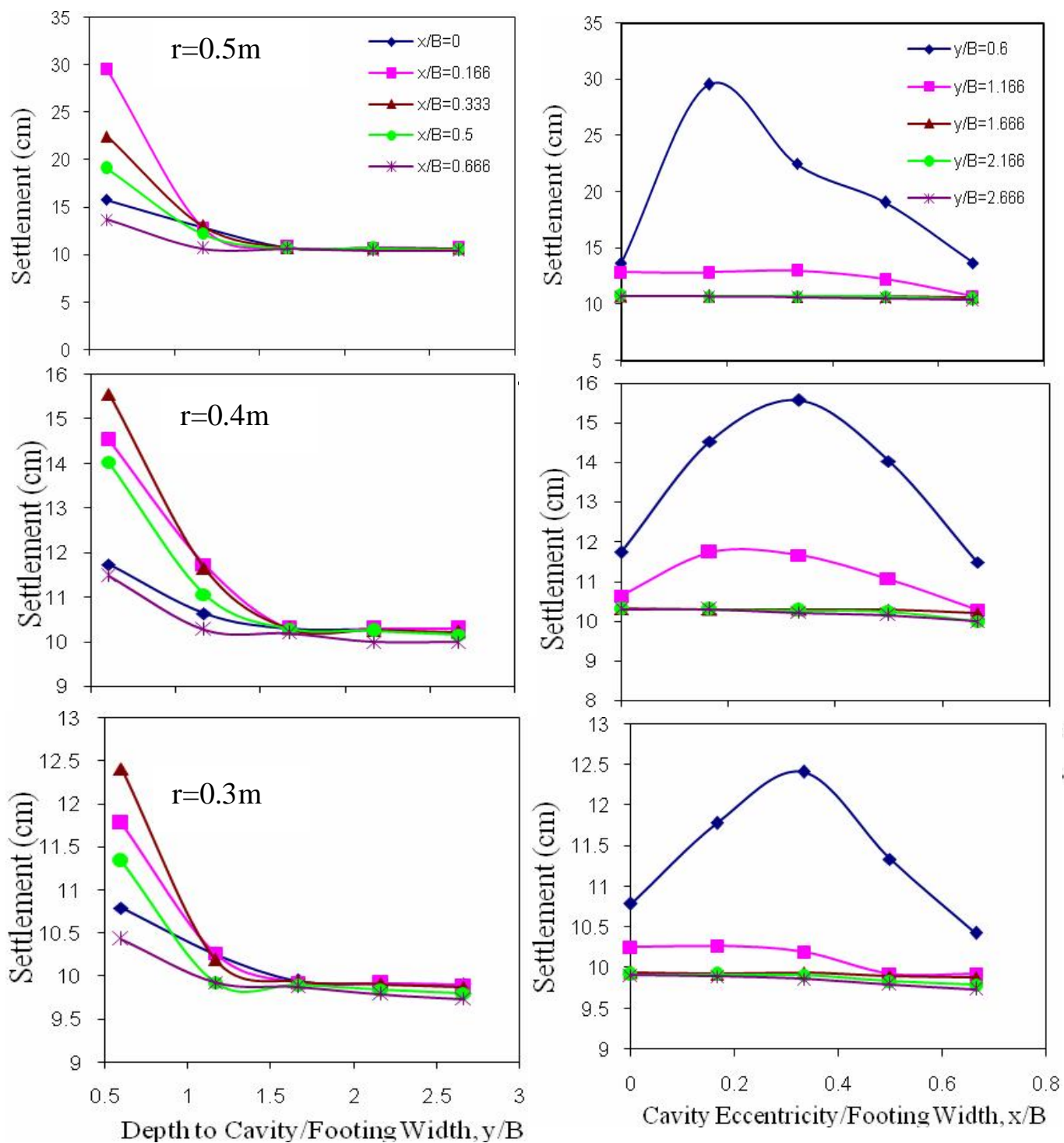

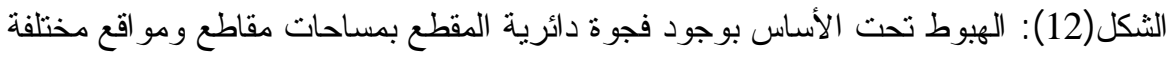

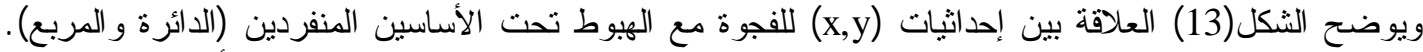

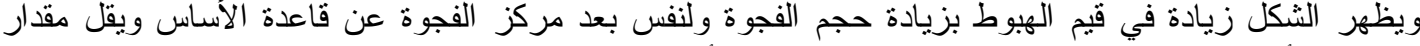

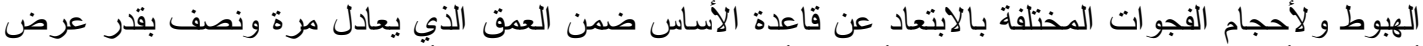

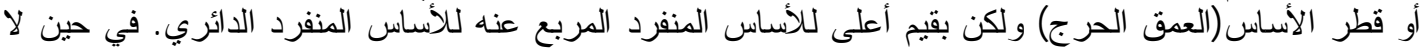

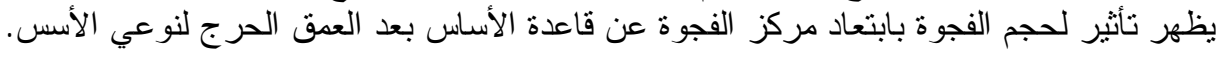



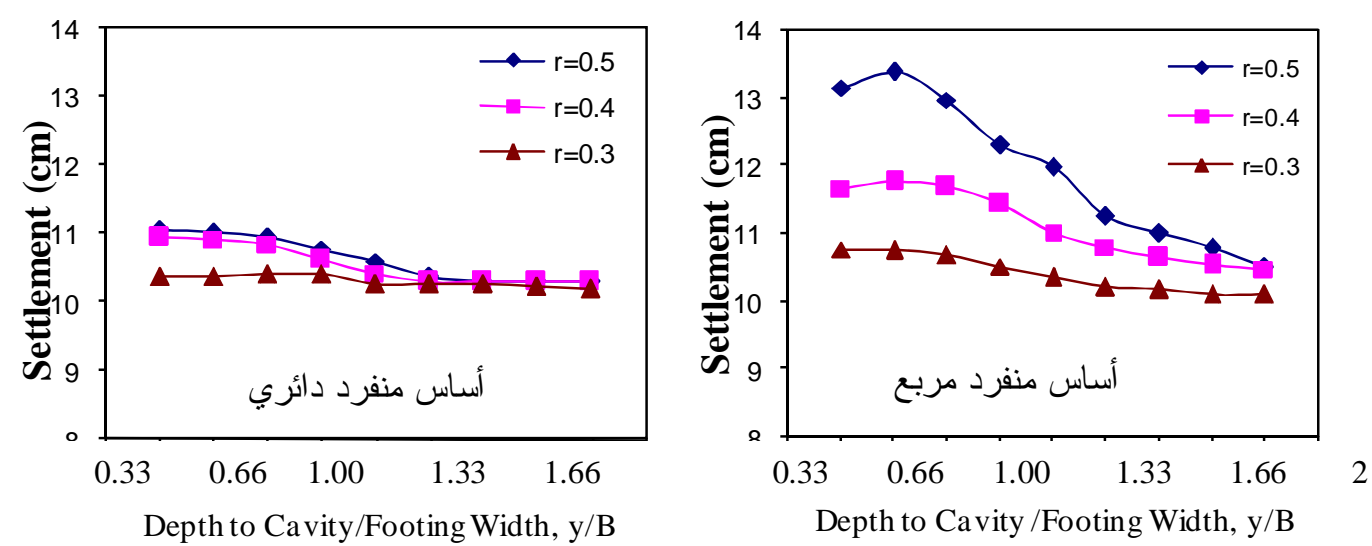

$$
\text { الثكل(13): علاقة بعد مركز الفجوة عن قاعدة الأساس مع الهبوط }
$$

من جهة أخرى تبين النتائج في الأشكال (14) و الأشكال(15) تأثثر زيادة حجم مقطع الفجوة على نزكيز الإجهاد

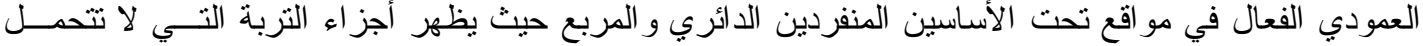

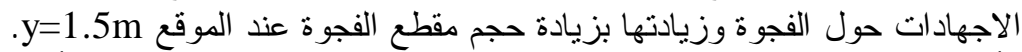
و أخير ا يمكن القول إن هناللك فرقا في قيم الإجهاد العمودي المسجل تحت الإدت الأساسين وبقيم أعلى تحــت الأســاس المنفرد المربع منه تحت الأساس المنفرد الد فئر الأري.
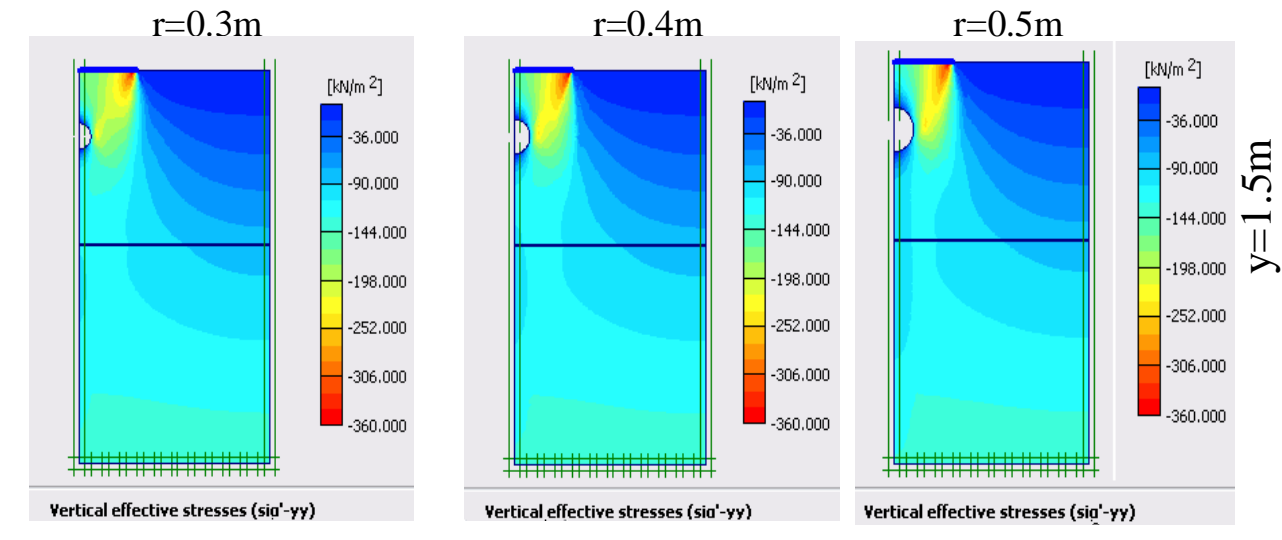

الثكل(14): تأثثر تغير حجم الفجوة على شدة نوزيع الإجهاد في التربة تحت أساس دائري منفرد

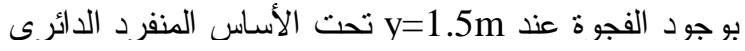

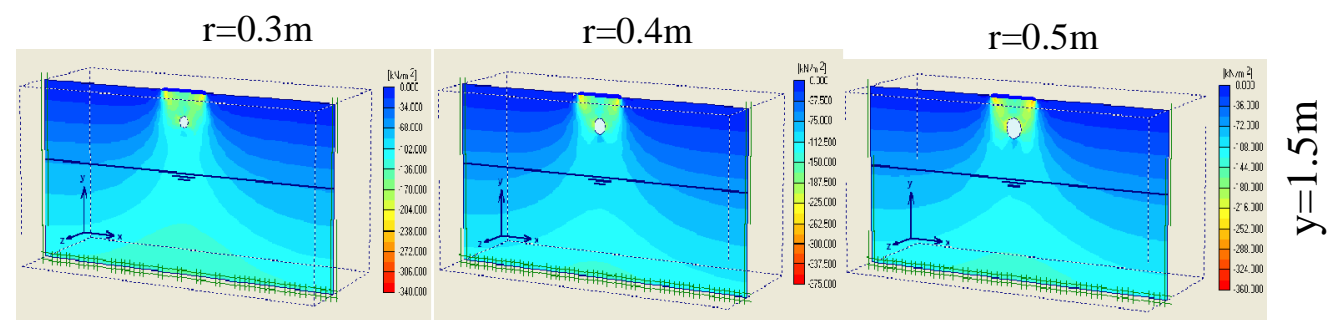

الثكل(15): تأثير تغير حجم الفجوة على شدة توزيع الإجهاد في التربة بوجود الفجوة عند y=1.5m تحت

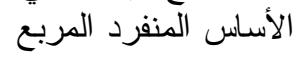

عمق وموقع الفجوة تحت الأسساس: تمت در اسة تأثنير عمق وموقع الفجوة تحــت أنسـواع الأسـس المختــارة.

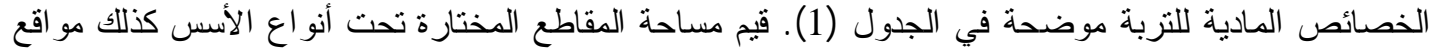
وبعد مركز الفجوة عن قاعدة الأساس موضح في الجدول(2). تم تغيير خصائص التربة في الدراسة الأجل التهن التمكن 


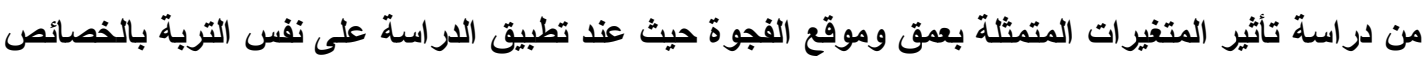

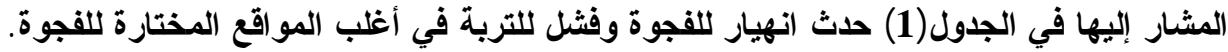

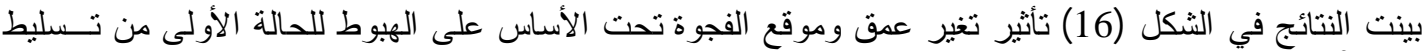

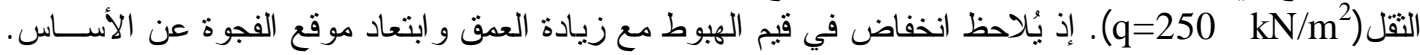

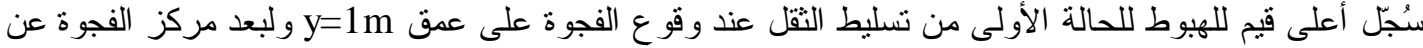

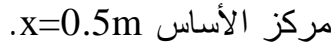

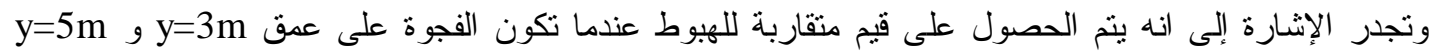
كما يقل تأثير وجود الفجوة على الهيوط عندما يكون مركز الفجوة على بعد 0.5m عن نهاية زاوية الأساس

. $(\mathrm{x}=1.5 \mathrm{~m})$

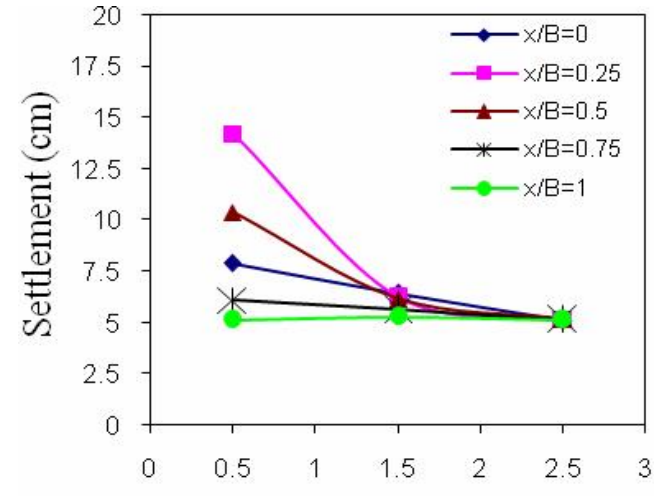

Depth to Cavity/Footing Width, $\mathrm{y} / \mathrm{B}$

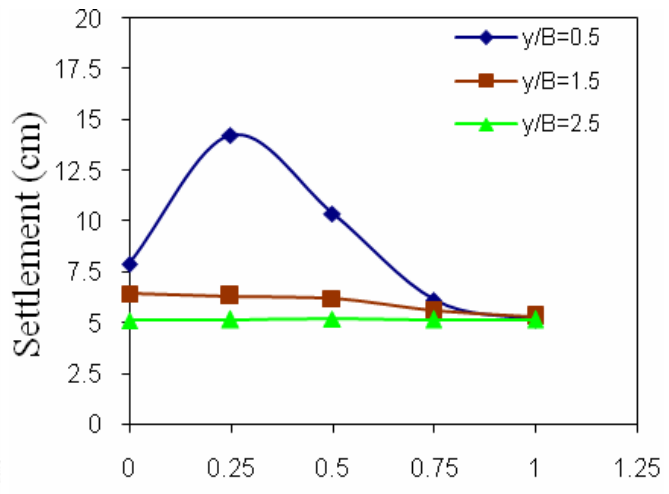

Cavity Eccentricity/Footing Width, x/B

الثكل (16) تأثير تغير بعد مركز الفجوة عن قاعدة الأساس الثريطي وبعد مركز الفجوة

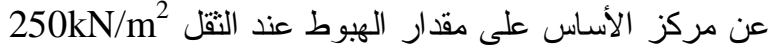

يظهر الثكل(17) عرضا آخر لنتائج الهبوط تحت الأساس بتغير بعد مركز الفجوة عن قاعدة الأساس وبعد مركز

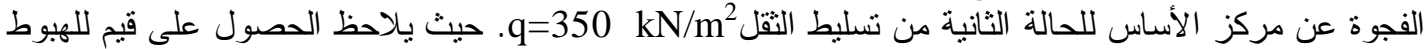

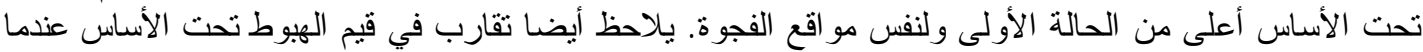

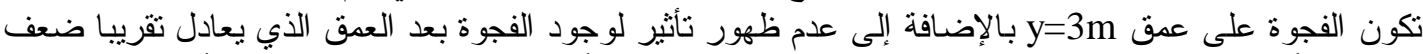

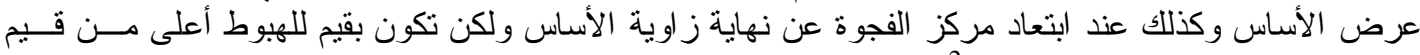

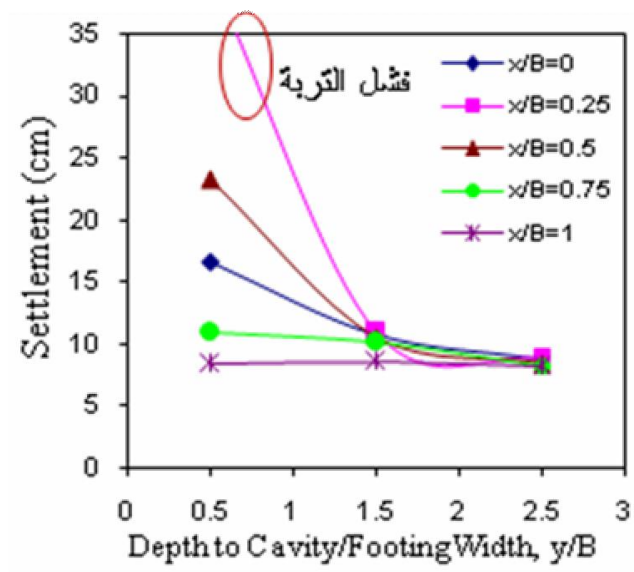

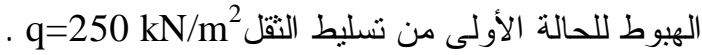

الشكل (17): نأثثير تغير بعد مركز الفجوة عن قاعدة الأساس الثريطي وبعد مركز الفجوة

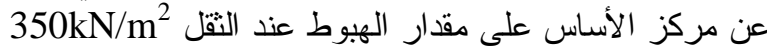




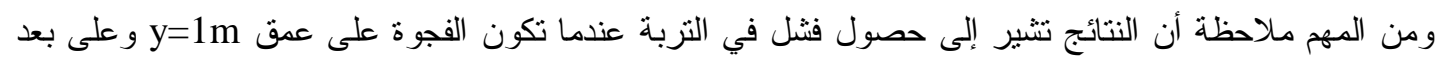

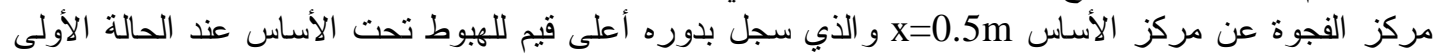
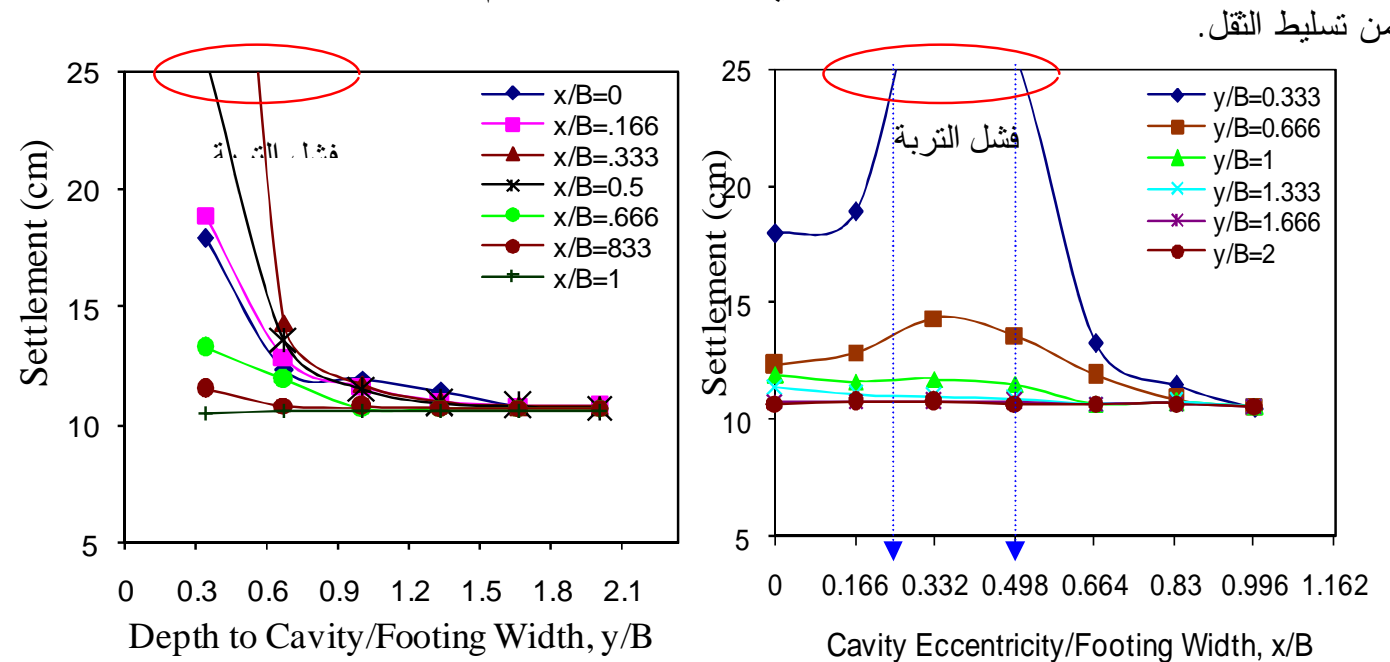

$$
\begin{aligned}
& \text { الثكل (18): العلاقة بين بعد مركز الفجوة عن قاعدة الأساس المنفرد المربع وبعد }
\end{aligned}
$$

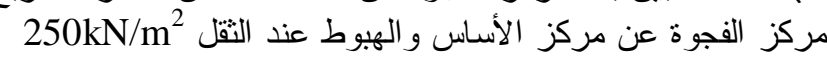

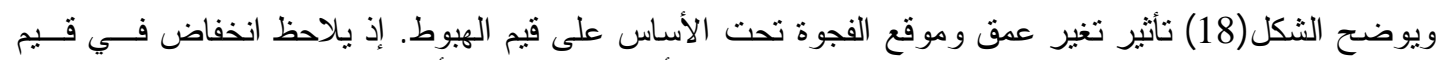

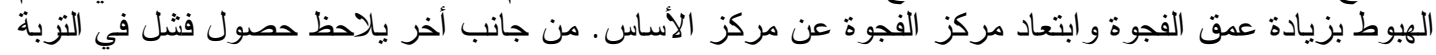

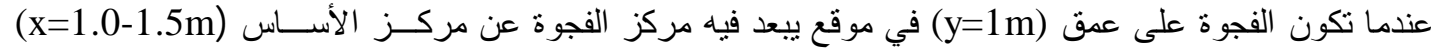

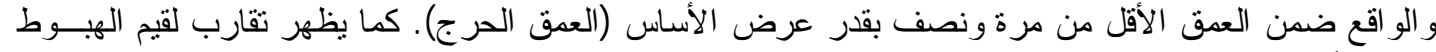

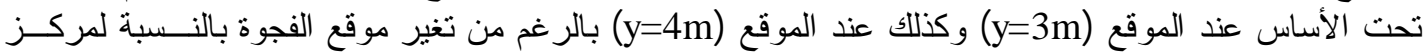

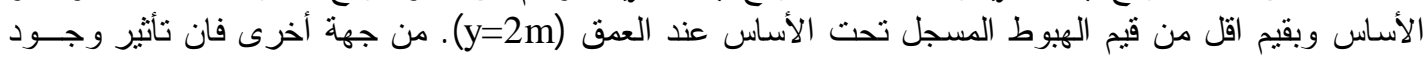

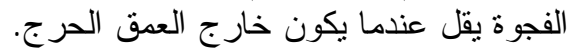
الثكل (19) عموما يُنشير إلى شكل نون نوزيع الإجهاد العمودي أسفل الأساس في التربة لحالتي النقل المسلط لحالة

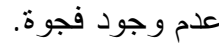

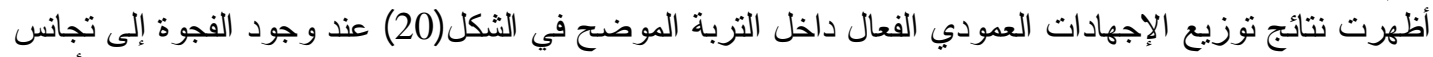

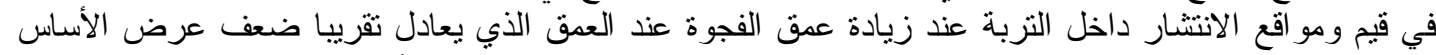

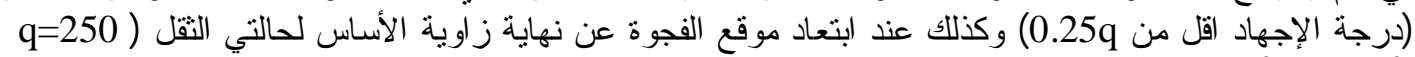
( q=350 kN/m $\mathrm{kN} / \mathrm{m}^{2}$
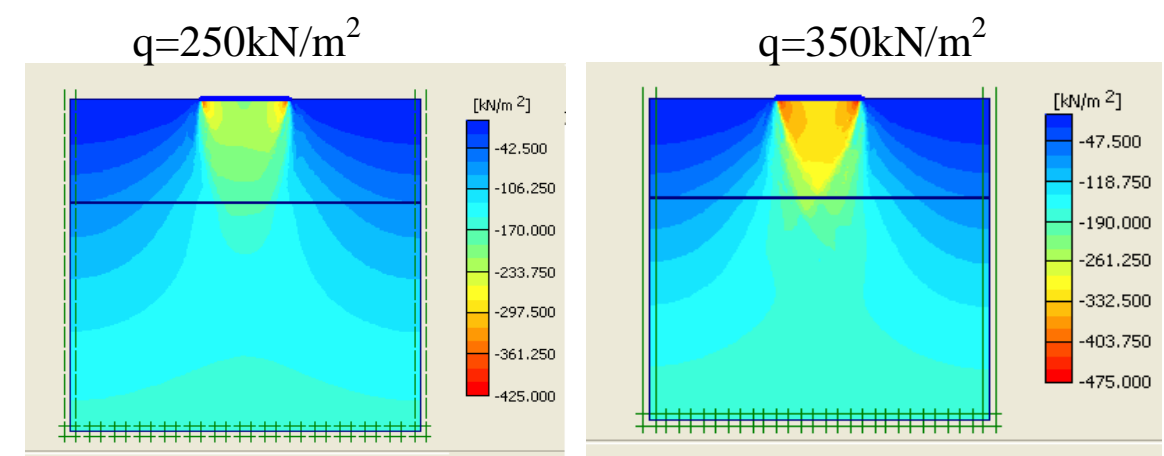

الثكل(19): شدة نوزيع الإجهاد في التربة بدون وجود الفجوة لحالتي التقل المسلط 


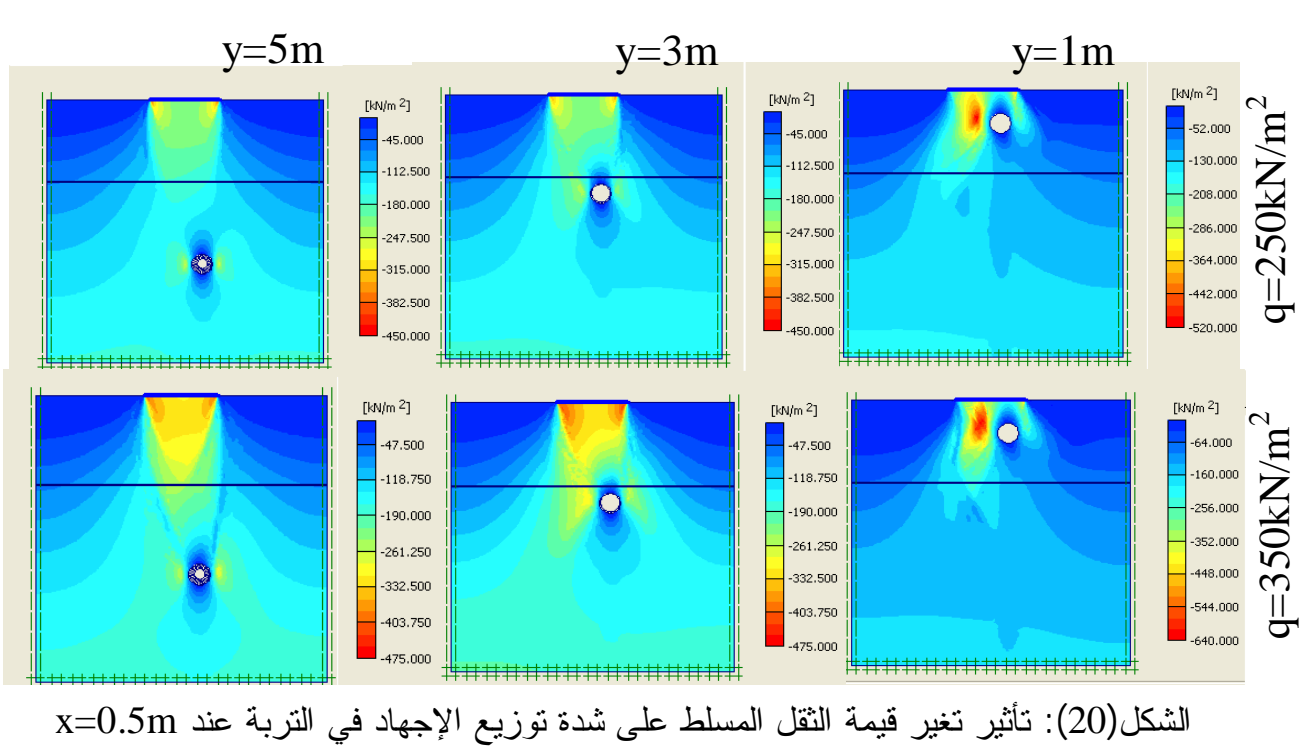

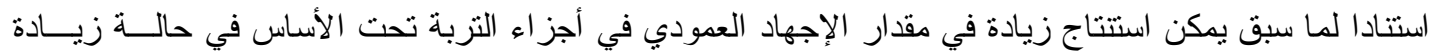

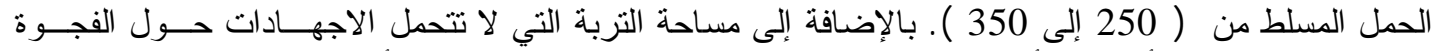

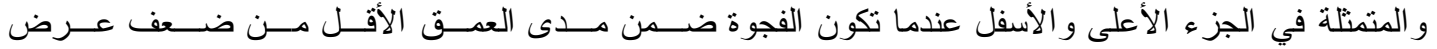

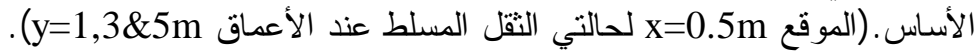

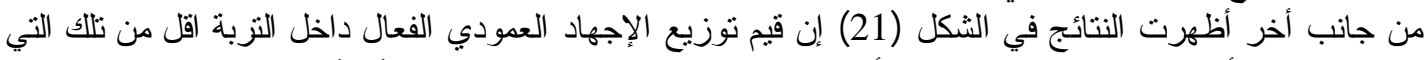

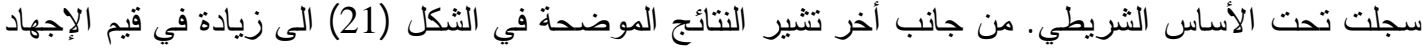

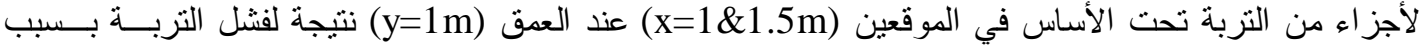

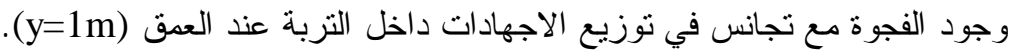

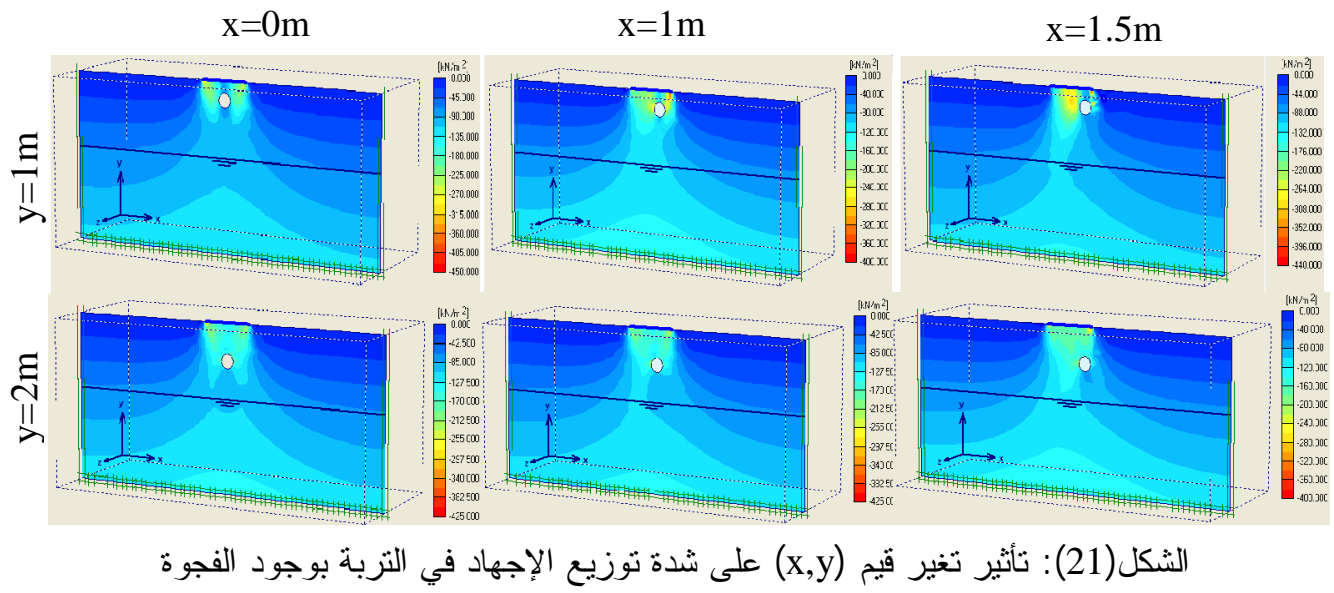

Conclusions الاستتناجات 4

من خلال إجر اء التحليل النظري بطريقة العناصر المحددة يمكن الوصول إلى الاستتناجات الآتية :

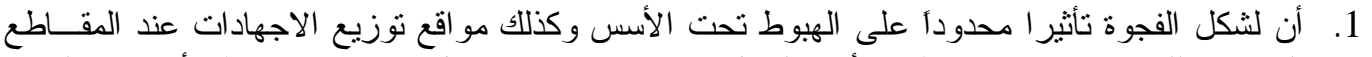

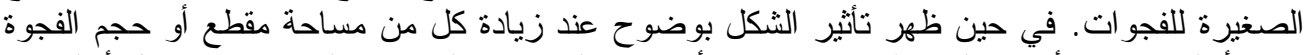

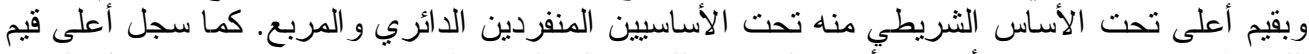

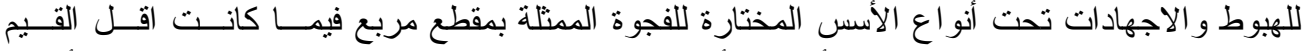

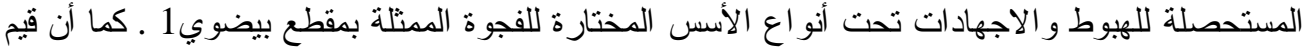

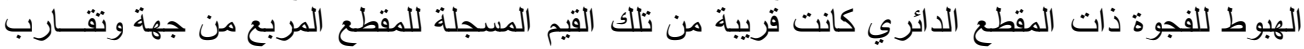

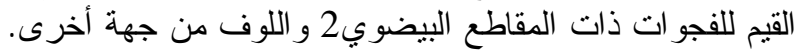




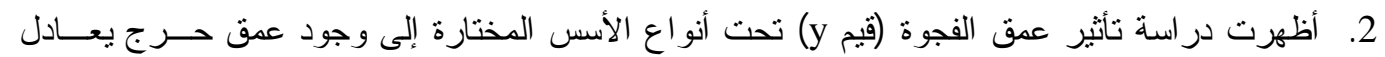

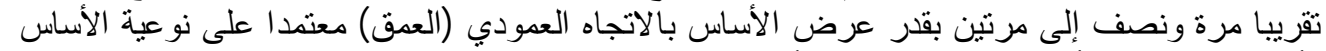

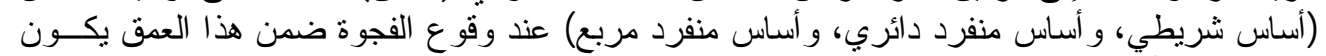

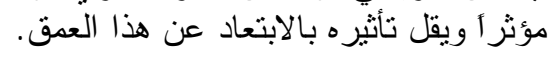

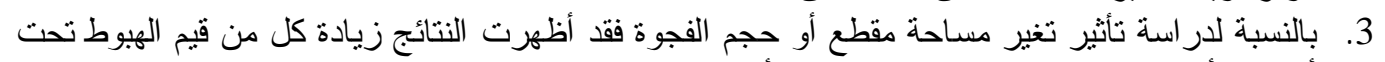

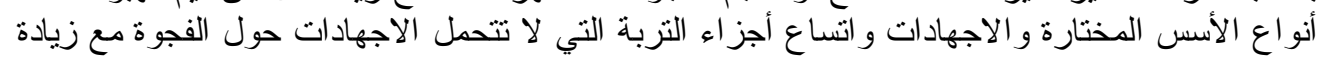

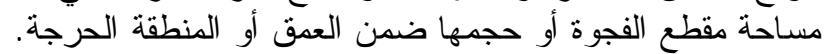

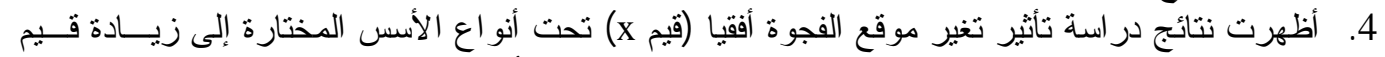

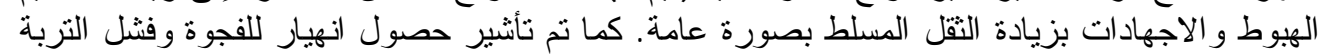

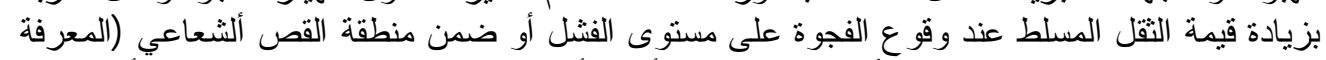

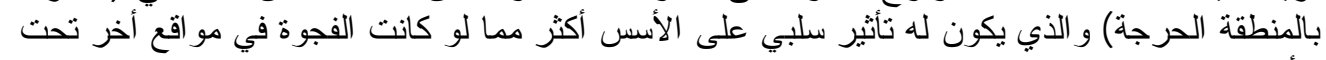
الأساس.

\section{-5}

1. AL-Kaashab, M. N., Thabet, K. M. \& AL-Ashow, M. O. (1986), "Some Effects of Cavities on the Stability of Buildings in Mosul City-A Case History", Jur. Geo. Soc., Iraq, Vol.19, No.3.

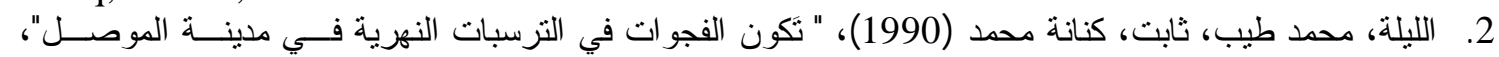

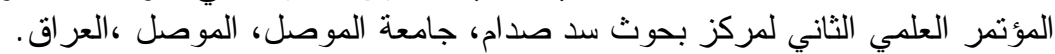

3. AL-Salim, T. H., \& Thabet, K. M. (2001), "Underground Cavities in AL-Mogher South Mosul City - Acase Study", Raf. Jour. Sci., Vol. 12, No. 3, pp 66-75.

4. AL-Mahjoob, M. M., AL-Dewachi, B. M., \& AL-Salim, T. H. (2006), "A Reconnaissance Detection of Caves and Subsurface Channels by Horizontal Electrical Profiting", Jur., Geo., Soc., Iraq, Vol. 6, No. 1, pp 64-73.

5.Culshaw, M. G., \& Waltham, A. C. (987),"Natural And Artificial Cavities as Engineering Hazards", Quarterly Journal of Engineering Geology and Hydrogeology, Vol. 20, pp 139150.

6. Vogelaar, B. B. (2001), "Cavity Detection", MSc. Thesis, Earth Sciences Department, Utrecht University, Netherlands.

7. Styles, P., McGrath, E., \& Cassidy, N. (2005),"The Use of Microgravity for Cavity Charaterization in Karstic Terrains ", Quarterly Journal of Engineering Geology and Hydrogeology, Vol. 38, pp 151-169.

8. Wang, M. C., Kim, Y. U, \& Jun, J. T. (2001), "Cavity Effect on Collapse Load of Strip Footings ", Proceedings of $15^{\text {th }}$ International Conference on Soil Mechanics and Geotechnical Engineering, CONF. 15, Vol. 1, pp 317-320.

9.Peng, F. L., Kiyosumi, M. Ohuchi, M., \& Kusakabe, O., (2006), "Cavity Effects on the Bearing Capacity of Footing Foundations and the Calculation Method", Journal of Geotechnical Engineering, ASCE, Vol. No., pp 50-57.

10. Said, F. M. (2005), "Finite Element Analysis of Soil Settlement Due to Salt Dissolution", M.Sc. Thesis, AL-Nahran University, Baghdad.

11. Brinkagreve, R.B. J. (2001), "Plaxis3D Tunnel Manuals", Published, A.A.Balkema. a member of Swets \& Zeitlinger.

12. Brinkagreve, R.B. J. (2002), "Plaxis2D Manuals", Published, A.A.Balkema. a member of Swets \& Zeitlinger.

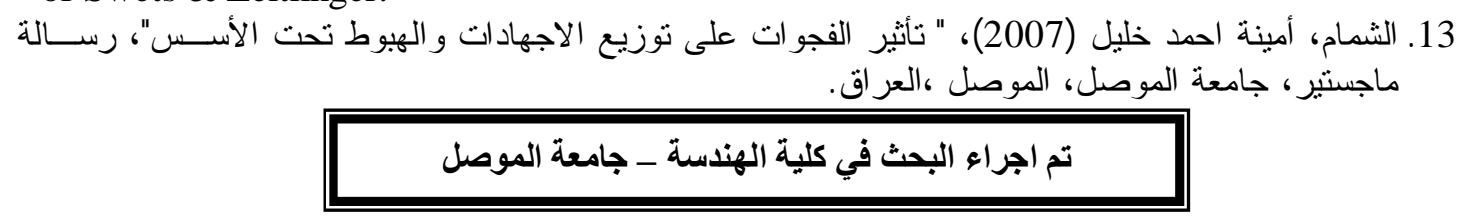

